\title{
Probabilistic stable motion planning with stability uncertainty for articulated vehicles on challenging terrains
}

\author{
Mohammad Norouzi $^{1}$ - Jaime Valls Miro ${ }^{1}$ - Gamini Dissanayake ${ }^{1}$
}

Received: 23 December 2014 / Accepted: 13 July 2015

(C) Springer Science+Business Media New York 2015

\begin{abstract}
A probabilistic stable motion planning strategy applicable to reconfigurable robots is presented in this paper. The methodology derives a novel statistical stability criterion from the cumulative distribution of a tip-over metric. The measure is dynamically updated with imprecise terrain information, localization and robot kinematics to plan safetyconstrained paths which simultaneously allow the widest possible visibility of the surroundings by simultaneously assuming highest feasible vantage robot configurations. The proposed probabilistic stability metric allows more conservative poses through areas with higher levels of uncertainty, while avoiding unnecessary caution in poses assumed at wellknown terrain sections. The implementation with the well known grid based $\mathrm{A}^{*}$ algorithm and also a sampling based RRT planner are presented. The validity of the proposed approach is evaluated with a multi-tracked robot fitted with a manipulator arm and a range camera using two challenging elevation terrains data sets: one obtained whilst operating the robot in a mock-up urban search and rescue arena, and the other from a publicly available dataset of a quasi-outdoor rover testing facility.
\end{abstract}

Keywords Probabilistic path planning - Uncertainty analysis · Tip-over stability · Mechanical reconfiguration . Rescue robotics

Mohammad Norouzi mh.norouzi@gmail.com

Jaime Valls Miro

jaime.vallsmiro@uts.edu.au

Gamini Dissanayake

gamini.dissanayake@uts.edu.au

1 Faculty of Engineering and IT, University of Technology, Sydney (UTS), 15 Broadway, Ultimo, NSW 2007, Australia

\section{Introduction and related work}

The demand for autonomous robots in industry and field application is increasing with the technological advances in modern sensors, actuators, hardware and software facilities which make employing of robotics technology more economical and feasible. In field application, mobile robots are required to operate fully or semi-autonomously in harsh, unstructured environments such as agriculture (Santosh et al. 2014), mining (SeungBeum et al. 2014), planetary exploration (Liang et al. 2013) and search and rescue (Keiji et al. 2013) missions for example. The robot model used to validate the results of this work is the multi-tracked iRobot Packbot robot depicted in Fig. 1. The robot is equipped with multiple sensors to get feedback from its own kinematic and gather and analyse environmental data. Dealing with uncertainty about the effects of imperfect actuators and poor environmental sensor information is a very common challenging problem in navigation over rough terrains.

Although uncertainty is usually ignored in classical motion planning techniques (LaValle 2006), more up to date algorithms have investigated different approaches to take into account imperfect robot motion or sensing models (Sebastian et al. 2005). One of the well studied approaches developed in the literature to explicitly deal with uncertainties in the input data and system model parameters is the partially observable Markov decision process (POMDP) (Matthijs and Nikos 2005; Brooks et al. 2006). For example a POMDP model for finding belief-feedback policies for a team of robots cooperating to extinguish a spreading fire is presented in Candido et al. (2010). The proposed planning algorithm is able to employ user-supplied domain knowledge for the synthesis of information feedback policies.

A linear-quadratic Gaussian motion planning (LQG-MP) strategy that is able to take into account the motion and sens- 


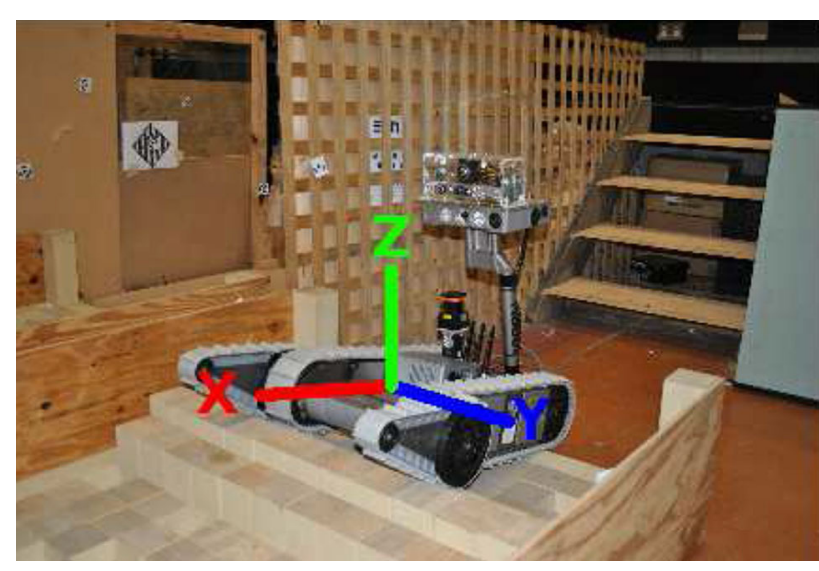

Fig. 1 The iRobot Packbot robot with a $1 \mathrm{DoF}$ arm, pan-tilt sensor unit and two flippers on a mock-up USAR arena

ing uncertainty is illustrated in Berg et al. (2011). Assuming a Gaussian model of uncertainty and having a linear-quadratic controller, the LQG-MP method aims to characterise priori probability distributions of the state of the robot in advance. The performance of LQG-MP is studied using simulation experiments where the rapidly exploring random tree (RRT) (LaValle 1998) is employed to generate the candidate paths. Motion planning in dynamic uncertain environments is another challenge for mobile robots operating in close proximity with many other moving agents; e.g. a service robot acting as a waiter in a restaurant, or mobile robots in exhibitions and trade fairs. In this case, the future evolution and uncertainties of the states of the moving agents and obstacles also needs to be addressed. A strategy to account for future information gathering in the planning in dynamic, uncertain environments is presented in Toit and Burdick (2012). The uncertainty in location of the robot and obstacles is considered using a partially closed-loop receding horizon control algorithm that is able to integrate the prediction, estimation, and planning and approximately solve the stochastic dynamic programming problem.

The path following with uncertainty has also been studied by the control community. A Kalman-based active observer controller for the path following of wheeled mobile robots subject to non-holonomic constraints is presented in Coelho and Nunes (2005). The effect of external disturbances, general model errors, and uncertainties present in the system are reduced by adding an extra state (the "active state") to the controller design. The effectiveness of the proposed pathfollowing controller is evaluated via simulation results for a wheelchair robot following a straight line and a circular path. More recently, a path following controller design approach for articulated manipulators based on transverse feedback linearisation is presented in Gill et al. (2013). The Lyapunov redesign (Parks 1966) method is employed to make the proposed controller robust against modelling uncertainty. Experimental results of a four DoF manipulator with a com- bination of revolute and linear actuated links are provided where the end-effector was set to move in a circular path.

The uncertainty in a system can be considered in two types of stochastic methods: non-deterministic (a boundary is assumed for uncertainties), and probabilistic (the uncertainties are described using probability distributions) (Toit and Burdick 2012). We are employing the stability uncertainty in a probabilistic formulation. Other authors have looked at the problem of non-deterministic incorporation of uncertainty at the planning stage, e.g. by considering variations in the $2.5 \mathrm{D}$ terrain elevation data and localisation errors, as described in Iagnemma and Dubowsky (2004) for an articulated wheeled mobile robot. The original force angle (FA) margin (Papadopoulos and Rey 1996) was employed to evaluate the stability of the rover in the elevation map, therefore the position of robot's centre of mass (CM) and the ground contact points (CPs) would be the essential inputs to calculate the safety margin. The CPs are assumed to be under the wheels and are calculated based on the robot's kinematic and its position over the elevation map. A conservative path planning approach is adopted that considers terrain measurement uncertainty, where a set of potential worst-case robot configurations at boundary locations in the terrain are examined to make sure that the vehicle would remain stable for a given arbitrary fixed variance in the elevation map. If any posture in this set is proven unstable, the corresponding location in the map will be regarded as untraversable. To address the localisation uncertainty for a given path, all points along the path within a distance proportional to the assumed robot localisation uncertainty are examined given all possible configurations. A point in the terrain would be considered as a feasible point for path finding purposes only if all configurations in the overall search have been proven to be stable. The output of this brute-force approach is a simple failure or success, with no concern for the probability of a tip-over instability.

A strategy for global path planning over ruggedised terrains while accounting for stability uncertainty is presented in this work. A novel safety confidence (SC) stability margin based on the conclusions of the statistical stability analysis technique described in Norouzi et al. (2013b) is introduced. The proposed probabilistic stability criterion is employed to advance further the deterministic stable path planning strategy described in Norouzi et al. (2013a), proven to be particularly suitable for search and rescue missions, with the goal of improving robot navigation safety in scenarios where the model of the system and the sensory data available to the robot may be imperfect. As also noted in that work, the proposed strategy is equally applicable to planning in large areas where prior knowledge of the terrain is assumed, or in exploratory settings where the robot needs to create the coverage map as it navigates further and only partial information from the surrounding area is available, hence setting goals in closer vicinity. 
The FA stability measure (Papadopoulos and Rey 2000) was employed in Norouzi et al. (2013a) to analyse the tip-over margin of the vehicle, and is also the choice in this work. It should be noted that there are several other criteria which can be combined using multi-objective optimisation in order to navigate in irregular terrains, e.g. when maximising ground clearance for more general wheel-legged mobile robots (Freitas et al. 2010). Considering further mobility criterion could indeed expand the applicability of the proposed planning method. The FA measure is a deterministic criterion that can be calculated based on the position of the robot's CM and the CPs interaction with the terrain, which form a convex area called "support polygon" (SP). As will be shown, the main difficulty in path planning using a deterministic constant stability margins is that a conservative large tip-over criterion can produce safe paths, but it may also easily end up being overly restrictive, and filtering out many probable pathways. On the other hand, planning on the tip-over stability margin boundary may clearly jeopardise stability if uncertainties are present. The main advantage of employing dynamic SC measure to path planning is that it can take into consideration the model uncertainties when finding paths, instead of resorting to restrictive fixed minimum safety margins. Moreover, while in Norouzi et al. (2013a) the mechanisms where provided to exploit stability both as a constraint and also as an added cost to the $\mathrm{A}^{*}$ (Hart et al. 1968) search optimisation process, in the overall path planning strategy proposed here we take the stand that simply using it as a constraint is appropriate to guarantee paths that are "confidently" stable. In essence we are advocating for the fact that so long as we are confident the final path found will be stable, it is less relevant whether another one might be slightly more stable, as that's ultimately less relevant to the final outcomes in a realistic setting, and we suggest not spend computational resources in doing that.

The effectiveness of the proposed probabilistic tip-over measure in stable path planning over challenging terrains is confirmed using a grid based $\mathrm{A}^{*}$ algorithm as well as a sampling based RRT planner. The model of the Packbot robot shown in Fig. 1 is imported to a dynamic physic simulator engine and comprehensive simulations in a USAR arena and data from a quasi-outdoor rover testing facility at the University of Toronto Institute for Aerospace Studies (UTIAS) (Tong et al. 2013) are provided. Part of this work was initially suggested in Norouzi et al. (2014) and has been hereby extended with further analysis and discussions, and its generalisation to another cost-based planner in the form of a randomized RRT planner.

\section{Overview of stability analysis}

The most common stability margins can be calculated based on two informations, the robot's CM and its SP defined by

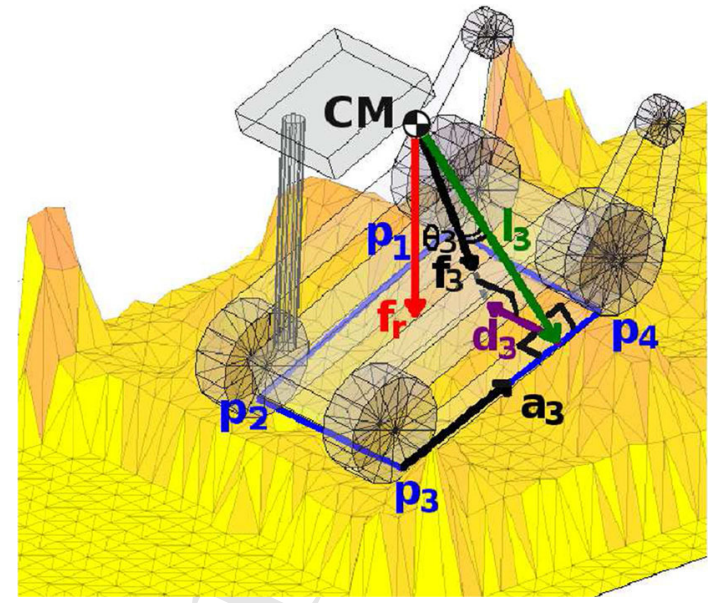

Fig. 2 The 3D FA stability measure for $n=4$ and $i=3$ i.e. for third axis of a SP with four CPs. The CM's position has been shifted up and vectors are scaled for easier visualization. The FA measure can be intuitively described as the effect of the net force and moment over CM projected on the SP e.g. $\beta_{3}=\theta_{3}\left\|\mathbf{d}_{\mathbf{3}}\right\|\left\|\mathbf{f}_{\mathbf{3}}\right\|$

the convex area spanned between the ground CPs. While the CM may be easily evaluated from the robot's kinematic state, prediction of SP is not a trivial problem and some works like (Liu and Liu 2010) have considered an ideal support polygon (ISP) for the vehicle, i.e. the CPs are assumed to be fixed under the sprockets of the robot. It is illustrated through some experiments in Norouzi et al. (2013a) how this is a strong assumption for the case of highly unstructured terrains, where CPs can lay anywhere along the robot's track and in general describe a variable support polygon (VSP). In this work no ISP is assumed and the process to derive the contact support polygon of a robot on a terrain is also briefly presented in this section.

\subsection{Force angle stability margin}

The FA stability margin (Papadopoulos and Rey 2000) was principally proposed for mobile machines with manipulators operating in construction, mining, and forestry. FA was proven to be one of the most effective stability margins. For example, a combination of the FA stability measure with an artificial potential field to obtain the demanded actuator values was used in Besseron et al. (2008). This simple criterion can then be computed based principally on the minimum angle between the effective net force and the tip-over axis normal. The normalized FA measure will be between zero (borders of instability) to one (most stable configuration). Negative values of the FA measure for an axis indicate that occurring tip-over instability about that axis is in progress. As shown in Fig. 2, the criterion $\beta_{i}$ for the $i$ th tip-over axis $\mathbf{a}_{\mathbf{i}}$ can be principally described by

$\beta_{i}=\theta_{i} \quad\left\|\mathbf{d}_{\mathbf{i}}\right\|\left\|\mathbf{f}_{\mathbf{i}}\right\|, \quad i=\{1, \ldots, n c p\}$ 
where $n c p$ is the number of out-most CPs. $\mathbf{f}_{\mathbf{i}}$ is the component of effective net force $\mathbf{f}_{\mathbf{r}}$ which acts about the tip-over axis $\mathbf{a}_{\mathbf{i}}$. $\theta_{i}$ is the angle between $\mathbf{f}_{\mathbf{i}}$ and the tip-over axis normal $\mathbf{l}_{\mathbf{i}}$. $\mathbf{d}_{\mathbf{i}}$ is the minimum length vector from $\mathbf{a}_{\mathbf{i}}$ to $\mathbf{f}_{\mathbf{i}}$. For example in this work $\mathbf{a}_{1}, \mathbf{a}_{2}, \mathbf{a}_{3}$ and $\mathbf{a}_{4}$ are left, rear, right and front axis respectively as illustrated in Fig. 2. The angles are in reference to the support pattern, which is the convex polygon derived from the CPs of the robot, and are sensitive to changes in CM's height. The overall robot's FA measure $\beta$, is given by

$\beta=\min \left(\beta_{i}\right), \quad i=\{1, \ldots, n c p\}$

In general, mobile vehicles operate at low speed when travelling over rough terrain and quasi-static robot dynamics can be safely assumed (Iagnemma et al. 2003). Thus, the net force $\mathbf{f}_{\mathbf{r}}$ acting on the system's CM will come from the gravitational loading term

$\mathbf{f}_{\mathbf{r}}=\sum \mathbf{f}_{\text {grav }}=m_{\text {tot }} \mathbf{g}$

The control aspect of maintaining speed and dynamic stability along the path is not hereby considered given the scope of the work. As demonstrated by various practical results in the paper, a suitable low speed controller was developed that readily validated this assumption.

\subsection{Robot model}

Figure 1 shows the multi-tracked iRobot Packbot robot model and its coordinate frame convention that was employed in this paper to validate the simulation results. The mechanical structure consists of a skid-steer vehicle base, flippers (two synchronised small sub-tracks in the front) and an arm that carries a 2D pan-and-tilt unit equipped with several cameras and lights. It is clear that for these types of robots the arm and/or flippers angles $\left(\phi_{a}\right.$ and $\left.\phi_{f}\right)$ will significantly affect the location of the CM. Moreover, when the flippers are in contact with the terrain they change the shape of the SP, which in turn has a more significant effect on the stability of the robot.

\subsection{Robot posture reconfiguration}

The robot's posture between successive path points is updated using an analytically derivable reconfiguration objective function (Norouzi et al. 2013a). The cost function is able to address different objectives including sensor visibility, track-terrain interaction and energy expenditure in changing postures. There are in general a large number of conflicting objectives that can play a significant role when planning paths in the context of realistic scenarios. The stability of the robot remains, however, the critical constraint so that if robot is ever found to be unstable, the optimality of any other parameters should be scarified to always satisfy the stability margin.

The reconfiguration cost function of the robot $U_{c}$ is given by

$U_{c}=\sum_{i=1}^{n} U_{i}$

where $U_{i}$ represents the reconfiguration cost associated to the $i$ th joint. For the Packbot model used in this work $n=2$, i.e. the arm and flipper joints $\left(\phi_{a}, \phi_{f}\right)$. More details about the reconfiguration algorithm, robot's kinematic model and the effect of the mass distribution can be found in Norouzi et al. (2013a).

\subsection{Contact points prediction and stability criteria}

The calculation of stability margins is predicated on calculating the projection of the robots geometric underside on the points defining the terrain underneath so as to derive the CPs. While straightforward geometry-based propositions can possibly be derived to find out CPs for simpler convex robots surfaces, this is not necessarily the case for more complicated shapes. A generic solution is proposed where the robot-terrain prediction algorithm is based on the mathematical description of the robot in the open dynamics engine (ODE) (Smith 2005), a widely used physical rigid body dynamics simulator. A 3D model of the terrain has been constructed from the ranging data measured with the RGB-D camera situated on the head of the robot. The CP derivation scheme is predicated on calculating the projection of the robot's geometric underside on the points defining the terrain underneath. Under the assumption of quasi-static equilibrium, the simulator predicts the behaviour of the robot under the influence of gravitational forces for a given pose and posture configuration to extract the SP. Some examples of the Packbot robot at various locations in a two-step field terrain model are given in Fig. 3 .

Given a rigid box sitting steadily on a hard rough ground surface, the number of CPs can not be less than three. An analogy can be established for instance with a rigid fourlegged table, where one leg of the table would be left in the air when sitting stably unless the terrain is flat, or soft, in which case it will be four. The FA margin calculations requires the out-most CPs, hence a maximum of four possible CPs are assigned to form the vertices of the SP even when the rigid body makes full contact with the surface, i.e. when the terrain across the wheel sprockets is flat such as in a ramp or stairs.

The Packbot robot is not a truly rigid model in that it is equipped with hard rubbery tracks which, albeit minimal, allow a bit of sag and deformation, effectively making larger contact with terrain surfaces, even in uneven hard surfaces. 
Fig. 3 Examples of support polygon shapes over two step-fields terrain models
This robot-terrain interaction behaviour is extremely difficult if not impossible to model accurately. Hence, for the ODE CP calculations, the point set located within an allowance distance set by the terrain mesh-grid resolution and the measured deformations of the rubbery tracks has been considered, and the out-most points selected as CP. The robot will thus be regarded stable at a given location if the resulting SP fulfils the following criteria: $n c p \geq 3$ and $\beta>\beta_{\min }$, where $\beta_{\min }$ is 0 or an arbitrary (positive) lower bound set for the stability margin. This process is described in more detail in Norouzi et al. (2013a).

\section{Uncertainty analysis method}

The probabilistic stability margin calculation, the definition of the proposed safety confidence and it's use in the context of path planning which form the novel contribution of this paper are described in the following sections.

\subsection{Transformation of means and covariance}

The probabilistic approach for uncertain stability analysis is detailed in Norouzi et al. (2013b). For completeness, this section will quickly summarise the aspects most relevant to the novel proposition in this work. The general problem can be expressed as follows: for a $\mathrm{n}$-dimensional input vector $\mathbf{x}$ with given mean $\hat{\mathbf{x}}$ and covariance $\mathbf{P}_{x x}$, what would be the mean $\hat{\mathbf{y}}$ and covariance $\mathbf{P}_{y y}$ of a m-dimensional random variable vector $\mathbf{y}$, where $\mathbf{y}$ is related to $\mathbf{x}$ by a non-linear transformation $\mathbf{y}=g[\mathbf{x}]$. For the system hereby considered, the arm and flipper angles $\left(\phi_{a}, \phi_{f}\right)$ that determine the posture of the robot, the 3D model of a given terrain and the robot's position on it constitute the input parameters, i.e. $\mathbf{x}_{37 \times 1}=$ $\left(\phi_{a}, \phi_{f}, r x, r y\right.$, yaw, $32 \times$ terrain sections $)$. The output vector includes a list with (up to) four CPs, the $\mathrm{CM}$ and the FA stability measure, i.e.

$$
\begin{gathered}
\mathbf{y}_{16 \times 1}=\left(4 \times\left(C P_{-} x, C P_{-} y, C P_{-} z\right),\right. \\
\left.\left(C M_{-} x, C M_{-} y, C M_{-} z\right), \beta\right) .
\end{gathered}
$$

Without loss of generality, expressions are shown for the case of four CPs, while as indicated in Sect. 2.4, the robot can also be stable with three CPs. In that case the dimension of $\mathbf{y}$ equals $13 \times 1$.

Given the highly non-linear nature of $g$ [.], Taylor series approximation (Greenberg 1998) and general error propagation (Siegwart and Nourbakhsh 2004) are not applicable to enumerate $\hat{\mathbf{y}}$ and $\mathbf{P}_{y y}$. Standard Monte Carlo (SMC) (Rubinstein and Reuven 1981) is a proven iterative algorithm to estimate probability density functions of a general system's output response from a large set of random inputs. Hence, by introducing perturbations to the input parameters, ODE simulations can be carried out and $\beta$ subsequently calculated. The tendency to bigger input sets to attain more accurate distributions makes SMC computationally expensive. The structured unscented transform (UT) (Julier and Uhlmann 2004) has been proposed in the literature to address this issue, and was employed in this work to speed up the transformation of means and covariances. The overall technique as applied to this work, summarised in Algorithm 1, intelligently simulates the SMC method by choosing a deterministic set of inputs instead of a vast random sample population. 


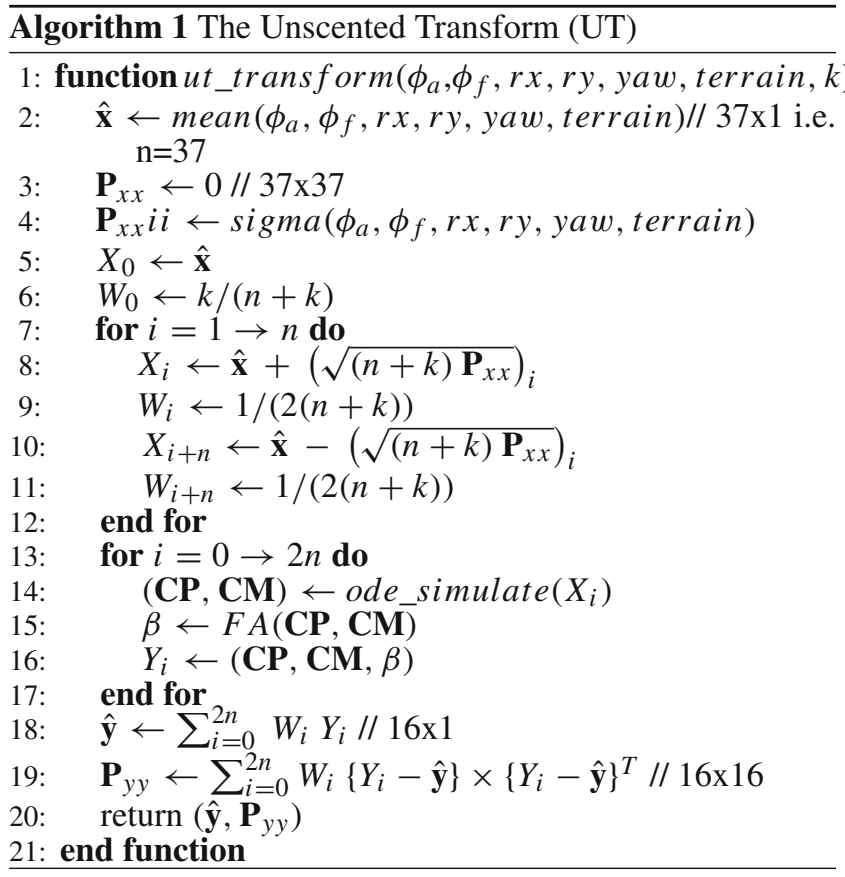

It is important to note that while only the mean and standard deviation of the FA distribution $\left(\beta_{\mu}, \beta_{\sigma}\right)$ are exploited for path planning purposes in this work, the output vector $\mathbf{y}$ also provides probabilistic information about the robot's CPs and CM. It is envisaged that it may well be possible to take advantage of these useful statistics in other stability margins, or for other purposes (e.g. computer graphics rendering applications).

\subsection{Probabilistic stability metric}

Assuming a standard normal distribution $N(0,1)$ for $\beta$, the cumulative distribution function $(\mathrm{CDF})$ is formulated as:

$\Phi(x)=\frac{1}{\sqrt{2 \pi}} \int_{-\infty}^{x} \mathrm{e}^{-\frac{t^{2}}{2}} \mathrm{~d} t$

This function describes the probability that $\beta$ will be found at a value less than or equal to $x$, where $\Phi(-\infty)=$ $0 \%, \Phi(0)=50 \%$ and $\Phi(\infty)=100 \%$. For a generic normal distribution $N\left(\mu, \sigma^{2}\right)$ for $\beta$, the cumulative distribution function can be transformed by

$F\left(x, \beta_{\mu}, \beta_{\sigma}\right)=\Phi\left(\frac{x-\beta_{\mu}}{\beta_{\sigma}}\right)$

Therefore $F\left(0, \beta_{\mu}, \beta_{\sigma}\right)$ will indicate the probability that $\beta$ will assume negative values (i.e. a tip-over is in progress). We can now define the SC margin to encapsulate our confidence in the stability prediction as

$S C(\beta)=\left(1-F\left(0, \beta_{\mu}, \beta_{\sigma}\right)\right) \times 100$
To intuitively understand the meaning of $S C$ the example in Fig. 4a is provided. The graph illustrates possible distributions for $\beta$, and the corresponding values for $S C$, based on three different robot postures at a given location on a terrain. Although the mean value of the green distribution is smaller than the blue one, a larger $S C$ value indicates more certainty in this configuration. A conservative fixed large $\beta$ will unnecessarily push the robot away from many potentially feasible trajectories. On the other hand, critically small safety margins may put the robot in jeopardy, particularly when traversing highly challenging terrains (e.g. stairs or rubble). By employing the proposed $S C$ margin instead, the system can benefit form a dynamic safety boundary that represents reliability in the output predictions.

For the special case when the mean value is exactly zero, the SC calculation would be independent of $\sigma^{2}(S C=50 \%$ always, as illustrated by Fig. 4b). In this case, although both distributions result in the same value for $S C$, for stability purposes a distribution with smaller $\sigma^{2}$ should be preferred (green curve in this example), indicating that the true $\beta$ is generally expected to be closer to zero and away from negative tip-over instability. Therefore, for the special case when $\mu=0, S C$ will be multiplied by $\left(1-\sigma^{2}\right)$ to lean towards configurations with smaller covariances. The following section provides some experimental results on maps obtained from a range camera fitted on the sensor head while the robot traverses over a ramp and a series of steps are presented that confirms the necessity and validity of the proposed probabilistic stability prediction method.

\subsection{Experimental results to prove the significance of a statistical approach for stability prediction}

To validate the results of statistical approach the robot was made to traverse over the actual ramp and hill step-field (HS) following a straight trajectory and constant reduced speed. A localiser running of odometry and 2D range data from an auto-levelled laser scanner was used to derive an estimate of the robot pose $(r x, r y, y a w)$ with a previously built 3D mesh of the arena, depicted in Fig. 9a. As the platform has got no suspension and the terrain is rigid, pitch and roll measurements from an on-board IMU can be assumed to be a veracious reflection of the vehicle's attitude when sitting on the terrain. The robot's pose $\left(\phi_{a}, \phi_{f}\right)$ was recorded from the actual on-board encoders during the experiments. The data from these tests was then analysed off-line to calculate the statistical properties of CPs and stability measures.

The inclination of the ramp illustrated in Fig. 5 is 30 degrees. The result of the ramp experiment is illustrated in Fig. 6. As shown in Fig. 6a, b, real inclination data is very close to that inferred by the simulator. The stability measure from a single simulation and mean value driven using UT in each point is depicted in black and red in Fig. 6d respectively. 


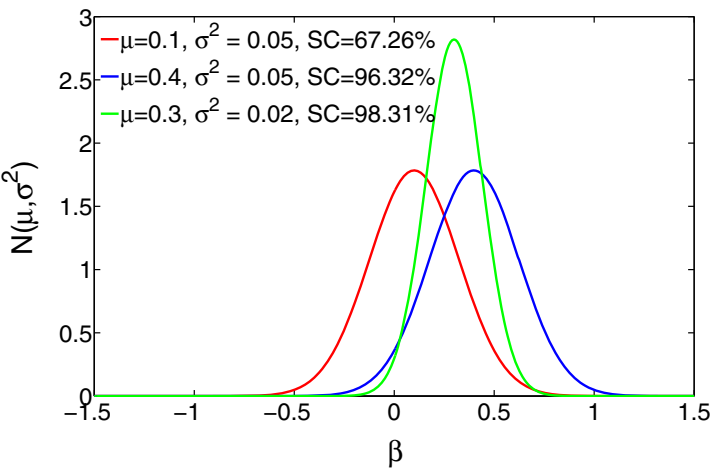

(a) $\beta_{\mu}>0$

Fig. 4 Example distributions for $\beta$ and the corresponding $S C$ values

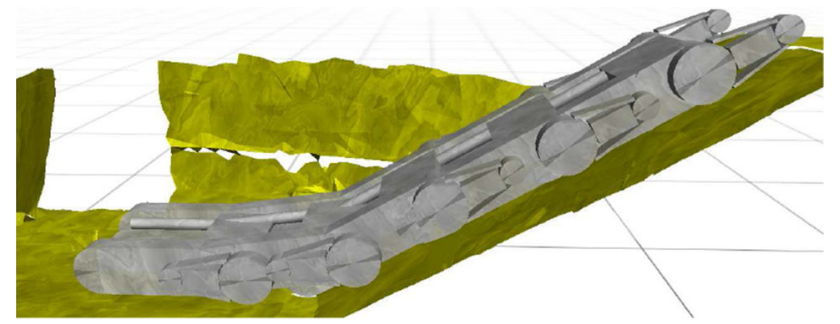

(a) Most stable.

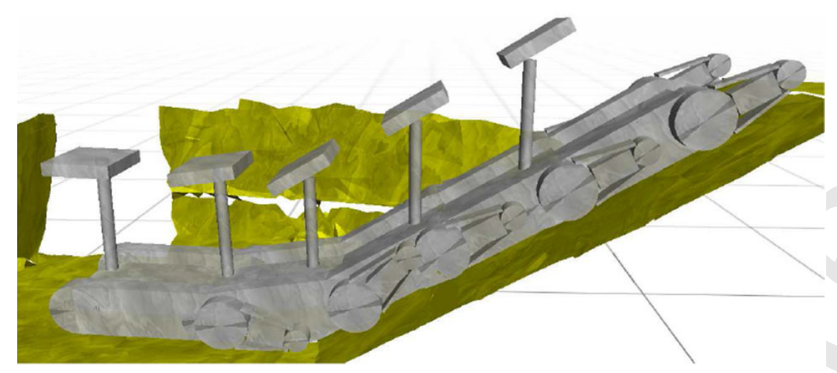

(b) Stable high-visibility.

Fig. 5 The side view of the robot configurations along the ramp (direction: left to right)

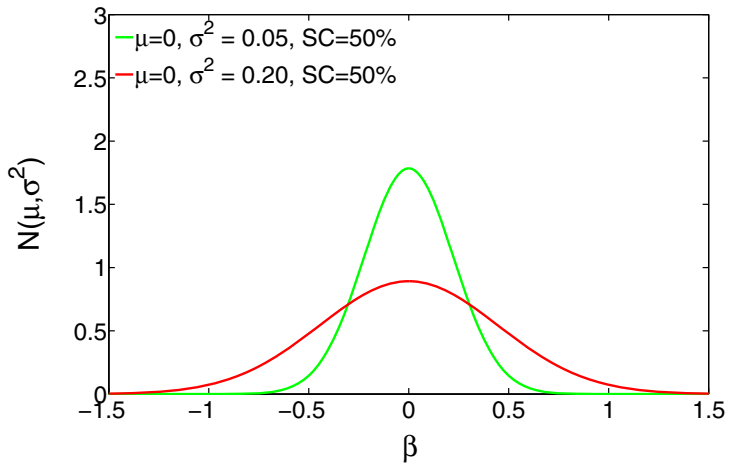

(b) $\beta_{\mu}=0$

case, the $\beta$ of the OSHV posture lies between the constant and the most stable stability margin. For safer posture trajectory the safety stability margin, $\beta_{\min }$ should be increased which will shift up the dashed black plot. The minimum value of $\beta$ in the most stable plot is around 0.4 , hence if the minimum $\beta$ in the planning was set to a value larger than this, the ramp trajectory would be regarded as unstable.

A side view of the path with the robot arrangements suggested by both planners are depicted in Fig. 5-omitted in some places to increase clarity. Comparing the results at the beginning of the ramp in Fig. 5a, $b$ shows that planning purely based on the stability margin has resulted in sudden flipper discontinuities, while the OSHV planner produced a soft and continuous kinematic trajectory thanks to the reconfiguration optimization between successive path nodes where joint discontinuities are penalised.

HS is an example to simulate common block obstacles, like rubble or unlevelled floors. The HS set-up illustrated in Fig. 1 (side view in Fig. 7) is composed of three successive $10 \mathrm{~cm}$ steps: two traversed "up", and one "down". The results of the experiment over the HS is illustrated in Fig. 8 in the same way as was earlier depicted for the ramp. As can be seen in Fig. 8a, b, the real inclination data is also closely captured by the simulator except at around 8 and $17 \mathrm{~s}$, when the robot tipped-over and had to be manually handled and returned to the HS to prevent a fatal crash. Although the calculated mean value for $\beta$ can be seen to be just positive over the path at those instances, $\sigma$ uncertainty analysis shows the robot tipping-over at those instances (when the crossing over the steps takes place).

Comparing these two examples shows that, despite the smaller inclination in the HS configuration, the robot is still more stable over the ramp than HS. Assuming that a fixed supporting-polygon and calculations of stability based on IMU data (like the approach in Roan et al. 2010) will lead to apparent stability, yet that is not the case. The traditional deterministic stability analysis method with variable supporting-polygon can be regarded as fairly reliable over 


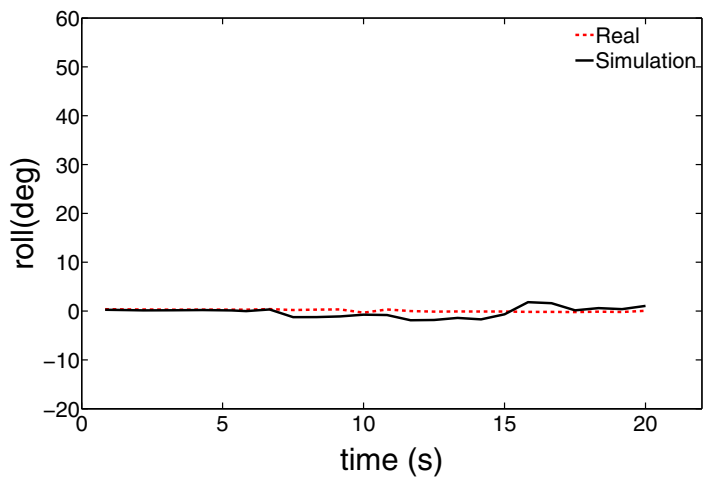

(a) roll.

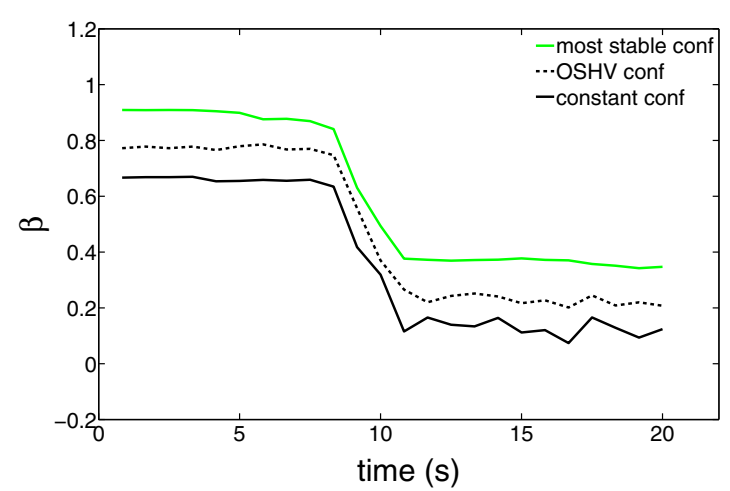

(c) Comparison of $\beta \mathrm{s}$.

Fig. 6 Experimental results over ramp

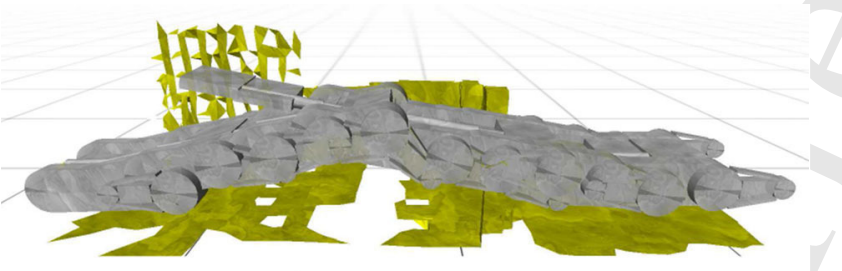

(a) Most stable.

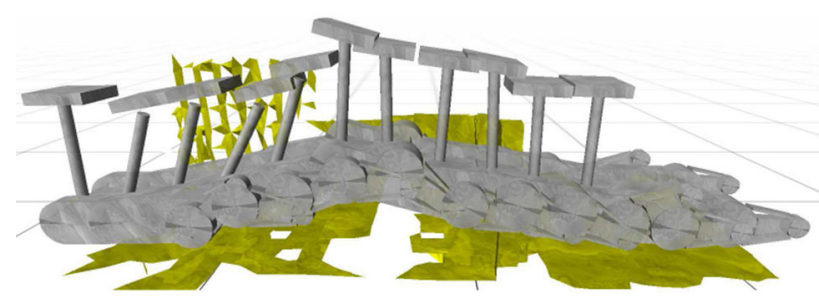

(b) Stable high-visibility.

Fig. 7 The side view of the robot configurations along the HS (direction: left to right)

simple topologies like ramps, but can't predict instability over more challenging obstacles like HS where the uncertainty in the input parameters can have a significant influence on the output stability metric.

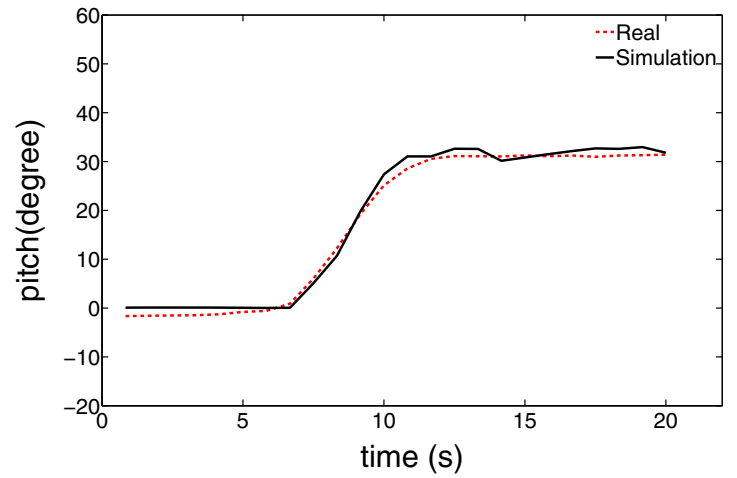

(b) pitch.

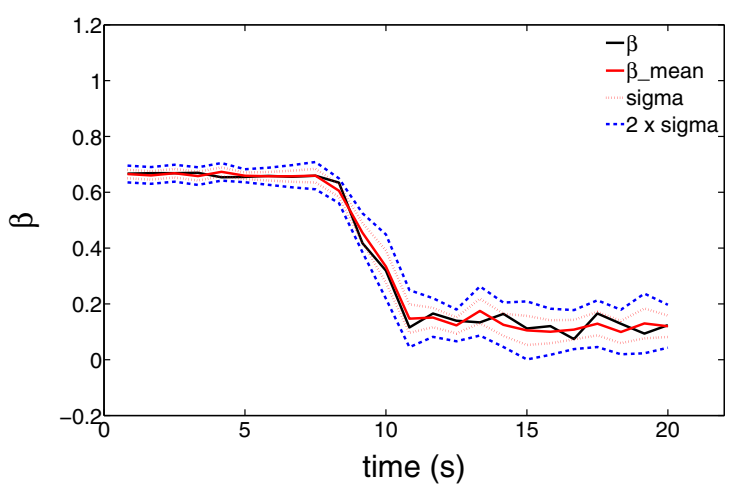

(d) Distribution of $\beta$.
In the same way, the patterns of $\beta$ acquired by three different configuration planning strategies along the same straight trajectory are illustrated in Fig. 8c. The solid black line is equal to the $\beta$ in Fig. $8 \mathrm{~d}$ and it is achieved while deriving the robot with a constant configuration $\left(\phi_{a}=90^{\circ}, \phi_{f}=45^{\circ}\right)$ and simulating the robot with recorded configuration and position over the 3D model of the terrain. For comparison purposes, the stability measures of the OSHV planner with $\beta_{\min }=0.2$ and the most stable configurations are depicted in dashed black and green respectively. It can be observed how for the OSHV posture $\beta$ is always smaller than the most stable stability margin. It can moreover be seen how in some places it is also smaller than the constant configuration's stability margin, as in that case there is no accounting for the additional visibility constraints in the robot pose. Thus in contrast to ramp traversing, at some places the constant configuration ends up marginally more stable than the calculated OSHV posture. Of course, for trajectories where increased safety posture is desired, $\beta_{\min }$ can be increased, effectively shifting the dashed black plot up so that it is always above the constant posture.

A side view of the path with the robot arrangements suggested by both planners is depicted in Fig. 7-omitted in some places to increase clarity. Comparing the results in 


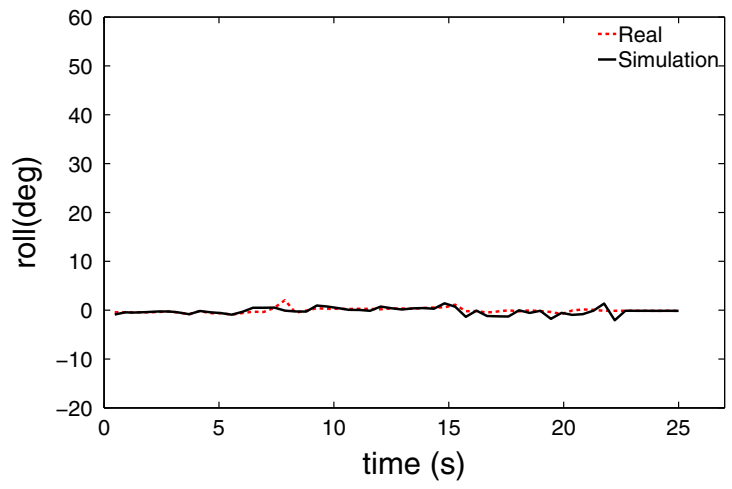

(a) roll.

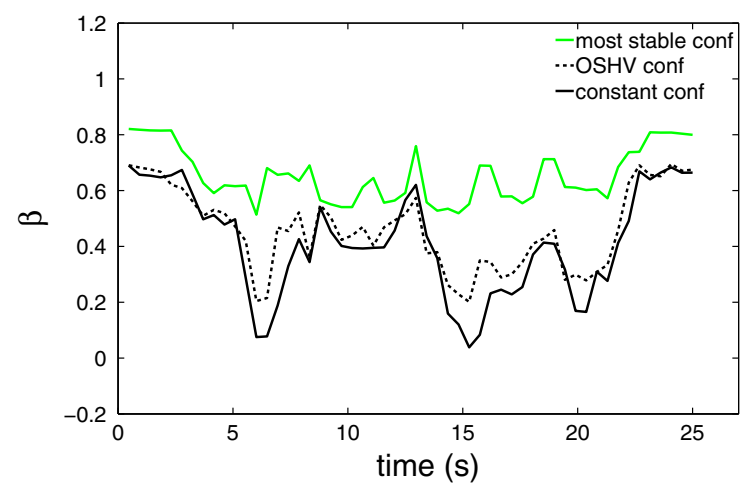

(c) Comparison of $\beta \mathrm{s}$.

Fig. 8 Experimental results over hill step-field

the beginning, middle and the end of the HS in Fig. 7a, b shows that planning purely based on the stability margin has resulted in sudden big changes for the flippers and arm while the OSHV planner produced a soft and continuous kinematic trajectory.

\section{Path planning with stability uncertainty}

To this end, this study has proposed the probabilistic stability measure $S C$ in Eq. 7 based on the cumulative distribution of the FA measure which indicates the probability that $\beta$ will be found to be positive. The following section illustrates the implementations with grid based $\mathrm{A}^{*}$ algorithm. The integration of the proposed strategy in a sampling-based RRT planner will also be presented in Sect. 4.3 for completeness. The effectiveness of the approach has been evaluated using two challenging terrain data sets, and then compared to the OSHV planner.

\subsection{Test arenas}

The USAR test arena is chosen to investigate the performance of the technique in an indoor setting with distinctive features

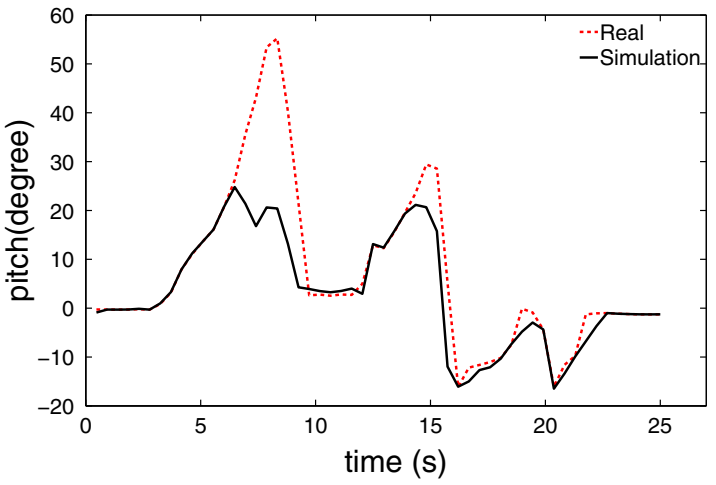

(b) pitch.

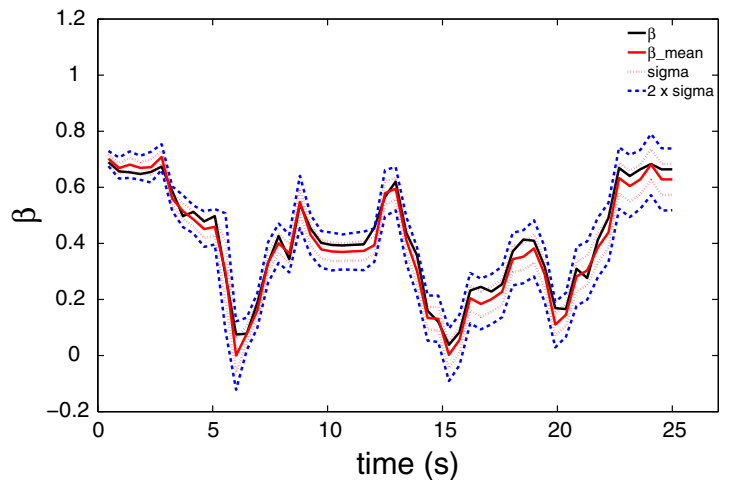

(d) Distribution of $\beta$. such as stairs, rubble etc., whereas the UTIAS arena is an example of a larger outdoor scenario. In both instances, the robot is expected to come up with configurations aimed at keeping the arm as high as possible to achieve the best possible field of view whilst satisfying the constraints imposed by the corresponding algorithms $\left(\beta_{\min }\right.$ or $\left.S C_{\min }\right)$.

The UTS mock-up rescue arena consists of a $6 m \times 8 m$ reconfigurable rectangle space with a ramp, a flight of stairs, open space and re-arrangeable blocks of step-fields. A small section is captured by Fig. 1. The 3D model of the terrain was built off-line by scan matching of the RGB-D data logs when manually operating the robot over the terrain at low speeds.

The UTIAS testing facility consists of a large dome structure, which covers a workspace area $40 \mathrm{~m}$ in diameter. These datasets are available online and for more information, the reader is referred to Tong et al. (2013). A grid resolution of $5 \mathrm{~cm}$ was assumed for both terrains which resulted in a 2D graph with dimensions of $164 \times 150$ and $784 \times 776$ for USAR and UTIAS arenas respectively. In order to make a fair comparison between the two planners a pre-processing step was first applied to both terrain models to label out obvious untraversable areas, e.g. walls and markedly steep slopes. 


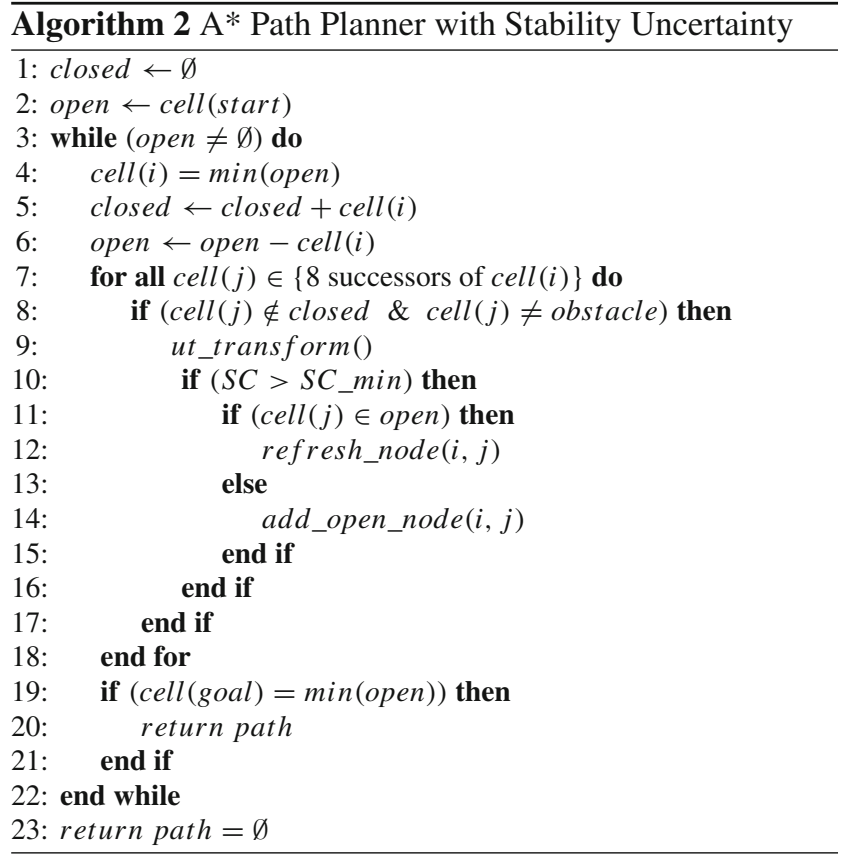

\subsection{Implementation with $A^{*}$ planner}

For comparison purposes, lets first briefly review the deterministic OSHV planner which was introduced in Norouzi et al. (2013a). The key contribution on this algorithm was the introduction of a stability constraint to a costbased planner. Essentially, the stable $A^{*}$ algorithm first examines the stability of the robot when opening a new search node at a new location with a given configuration. The node is considered stable if $\beta$ is larger than some nominal $\beta_{\min }$ that is satisfied. The present proposal, abstracted by Algorithm 2, takes into account $S C$ as described by Eq. 7 through the $u t$ transform() Algorithm 1, effectively transforming the fixed stability constraint $\left(\beta>\beta_{\text {min }}\right)$ into a minimum confidence threshold $(S C>$ $S C_{\text {min }}$ ) representative of the certainty in the stability prediction.

\subsubsection{Results of $A *$ planner in the USAR arena}

Two sets of experiments are studied of planning based on varying allowable boundaries for $\beta_{\min }$ and $S C_{\min }$ in order to highlight the advantages of the probabilistic approach in generating safer and more optimal posture planning.

In the first scenario, planners are set to find a path from the top left corner of the USAR arena with a minimum possible $\beta_{\text {min }}=0.05$ and $S C_{\text {min }}=50 \%$ to the goal at the bottom right corner. The value of $\beta_{\text {min }}=0.05$ was obtained experimentally as the border of stability when the robot was sitting on the $35^{\circ}$ ramp of the arena, with the nominal con-

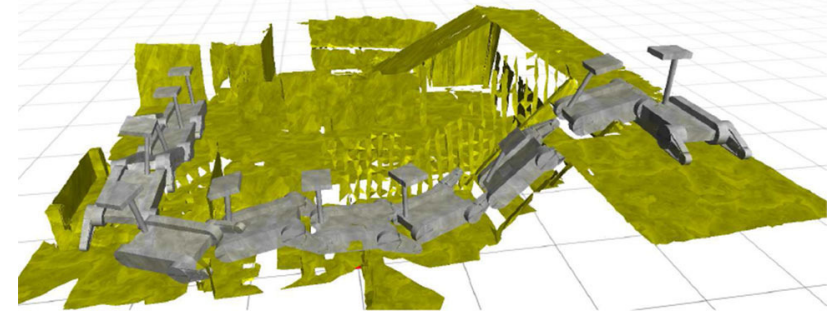

(a) Path with $\beta_{\min }=0.05$.

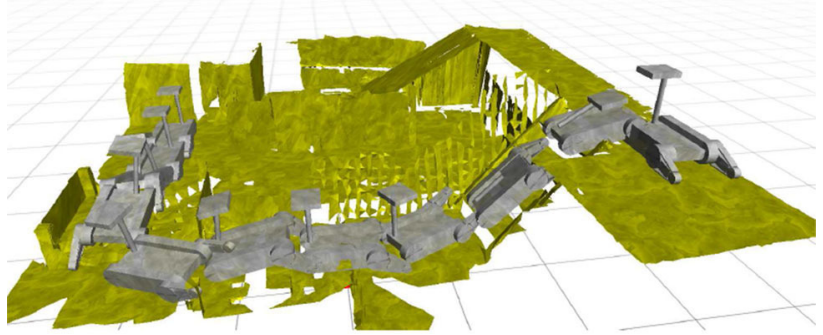

(b) Path with $S C \min =50 \%$.

Fig. 9 Planning based on the minimum safety margin and stability confidence in the USAR arena. Planning based on $S C$ generates safer postures over stairs $\left(\phi_{a}=0^{\circ}\right.$ in $\left.\mathbf{b}\right)$ when compared to the deterministic approach $\left(\phi_{a}=20^{\circ}\right.$ in $\left.\mathbf{a}\right)$

figuration $\left(\phi_{a}=90^{\circ}, \phi_{f}=90^{\circ}\right)$. A positive $\beta_{\mu}$ is the only requirement to achieve $S C_{\min }=50 \%$, consequently the minimum allowable safety confidence is assumed to be $50 \%$.

The results are depicted in Fig. 9, where Fig. 9a, b illustrate the outcomes of the shortest deterministic and probabilistically stable paths respectively. Only a limited number of the robot poses are shown in the figure for clarity. In both instances the final paths traverse through the step-fields and the stairs, and the robot configurations over both trajectories end up being quite similar (except on the stairs, way-points around 100-130 in Fig. 10, discussed below).

The comparison of $S C$ and $\beta$ over these trajectories are depicted in Fig. 10. The mean value of stability measure obtained using the UT transform $\beta_{\mu}$ at each instant is depicted in red, with the standard deviation $\sigma(68 \%)$ and $2 \times \sigma(95 \%)$ around the mean depicted in dashed red and blue in Fig. 10a, c. Figure 10b, d illustrate the corresponding $S C$ measures of the resulting two paths.

It can be seen how by setting an arbitrary lower boundary $\left(\beta_{\min }=0.05\right)$ the deterministic planner's limited concern about the instantaneous value of $\beta$ results in paths with instances where, although as shown in Fig. $10 \mathrm{a} \beta$ is computed to be always bigger than $\beta_{\text {min }}=0.05$, in some places the corresponding $\beta_{\mu}$ is actually negative $(S C<50 \%)$, indicates 
Fig. 10 Comparison of $S C$ and $\beta$ over the trajectories depicted in Fig. 9 where $\beta_{\text {min }}=0.05$ and $S C_{\min }=50 \%$ in the USAR arena. The horizontal dark green dash-dot lines are indicating the reference points where $\beta=0$ or $S C_{\min }=50 \%$

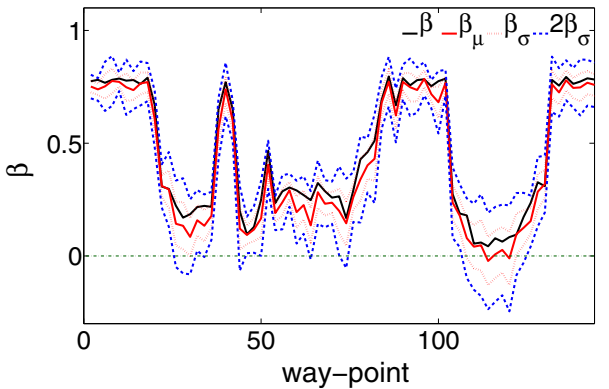

(a) $\beta\left(\beta_{\text {min }}=0.05\right)$.

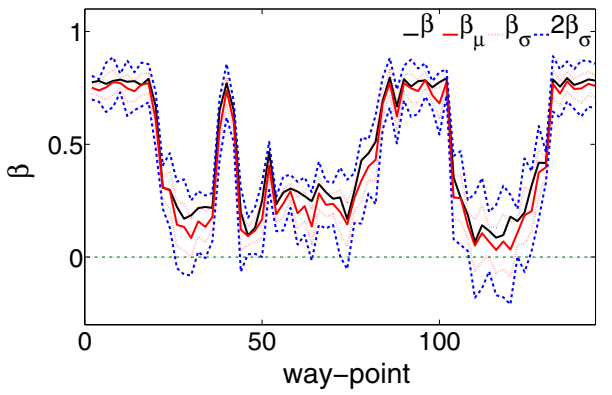

(c) $\beta\left(S C_{\min }=50 \%\right)$.

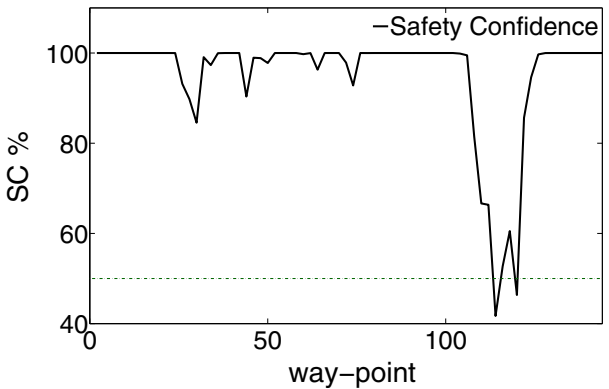

(b) $S C\left(\beta_{\min }=0.05\right)$.

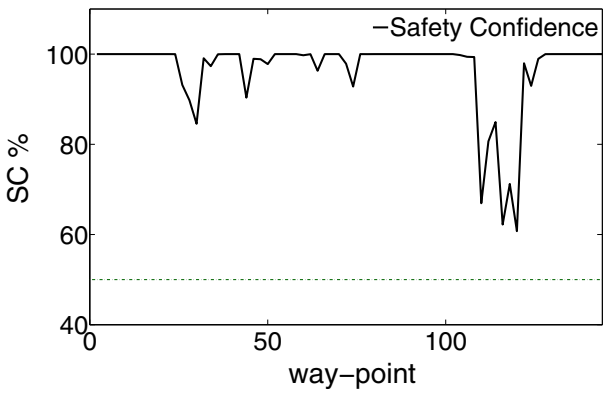

(d) $S C\left(S C_{\min }=50 \%\right)$.

a high risk for tip-over instability as illustrated in Fig. 10b. This happens for instance over the stairs (way-points around $117)$, where $\beta_{\mu}$ is indeed less than 0.05 .

On the other hand, as depicted by Fig. 10d, a planner considering an $S C_{\min }=50 \%$ might end up with instances when $\beta_{\mu}$ is less than 0.05 in some places (see Fig. 10c). However, $S C$ remaining over the threshold of $50 \%$ only requires a positive $\beta_{\mu}$, which is comfortably achieved by the planner generating postures with lower sensor head heights (e.g. $\phi_{a}=0^{\circ}$ over the stairs section depicted in Fig. 9b), compared to the resulting postures $\left(\phi_{a}=20^{\circ}\right)$ of a deterministic planner when $\beta_{\text {min }}=0.05$ (Fig. 9a). This example clearly shows how the probabilistic approach tends towards more conservative paths stability-wise than a deterministic planner in areas where uncertainty escalates.

In the following example the safety margin and stability confidence are increased to $\beta_{\min }=0.20$ and $S C_{\min }=70 \%$ respectively. Both criteria will now filter out the stairs and step-fields, tending towards a safer but longer path to the goal through the ramp, as shown in Fig. 11. Planning based on $\beta_{\min }=0.20$ has configured the robot with $\left(\phi_{a}=0^{\circ}\right)$ over the ramp. Yet given the higher certainty of the map over the ramp (as opposed to more rugged terrain sections), the probabilistic planner with $S C_{\min }=70 \%$ can satisfy the stability constraint with a better field of view configuration $\left(\phi_{a}=50^{\circ}\right)$ for the same area. As with the earlier example, the comparison of $S C$ and $\beta$ over the resulting trajectories are depicted in Fig. 12.

It can be observed how in the ramp area (way-points around $140-170)$ uncertainty is very small $\left(\beta_{\mu}\right.$ and covari-

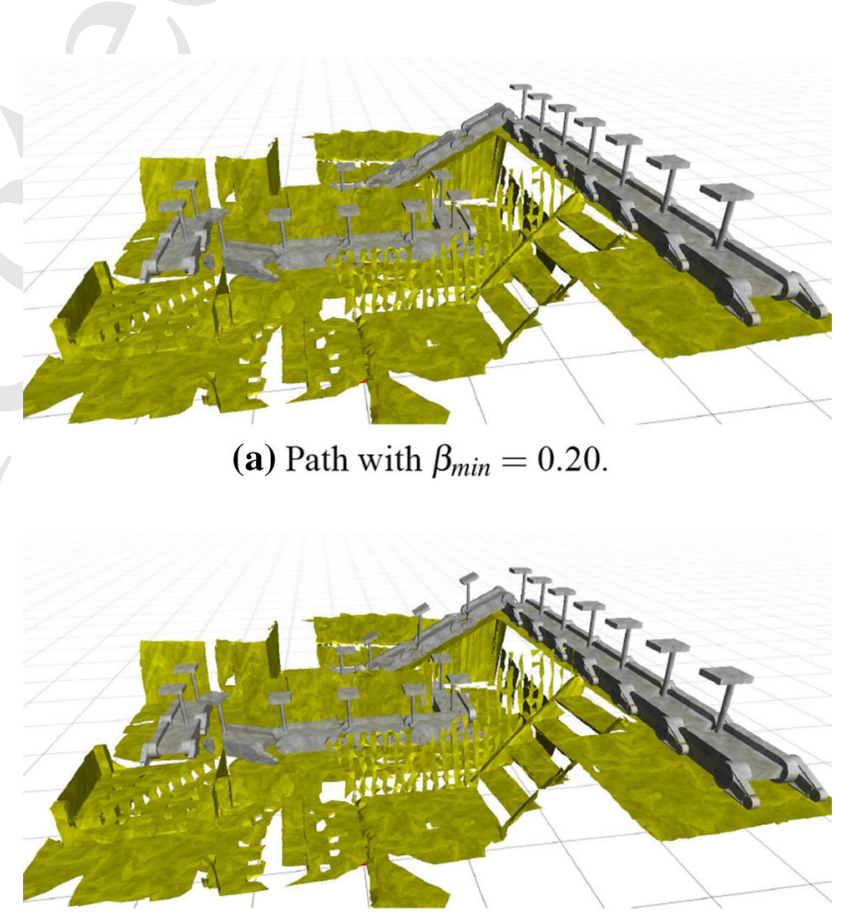

(b) Path with $S C \min =70 \%$.

Fig. 11 Planning based on a comfortable safety margin and stability confidence in the USAR arena. Planning based on $S C$ generates postures with better visibility over the $\operatorname{ramp}\left(\phi_{a}=50^{\circ}\right.$ in $\left.\mathbf{b}\right)$ compared to the deterministic approach $\left(\phi_{a}=0^{\circ}\right.$ in $\left.\mathbf{a}\right)$

ance around 0.20, Fig. 12a) and the probabilistic approach is then able to exploit this to generate postures with better visibility than the deterministic planner. 
Fig. 12 Comparison of $S C$ and $\beta$ over the trajectories depicted in Fig. 11 with $\beta_{\min }=0.20$ and $S C_{\min }=70 \%$ in the USAR arena

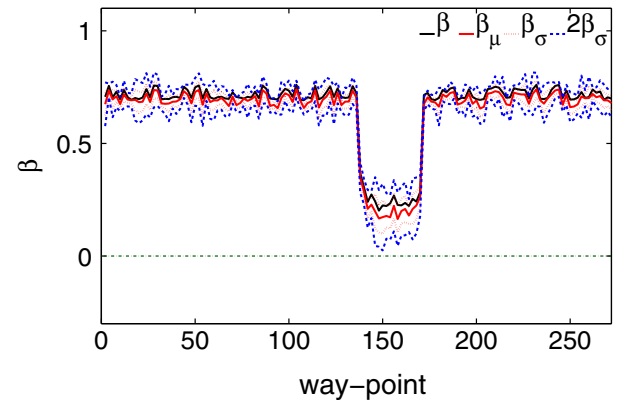

(a) $\beta\left(\beta_{\text {min }}=0.20\right)$.

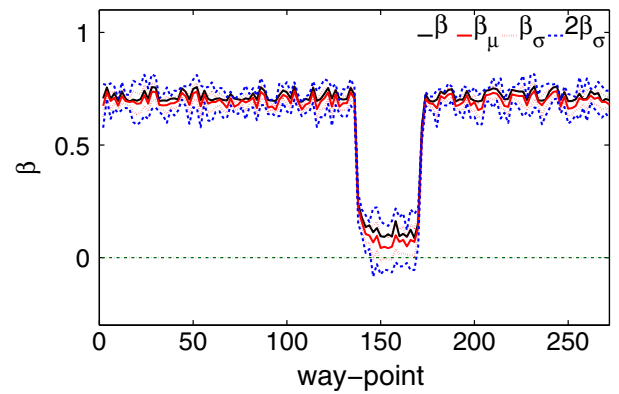

(c) $\beta\left(S C_{\min }=70 \%\right)$.

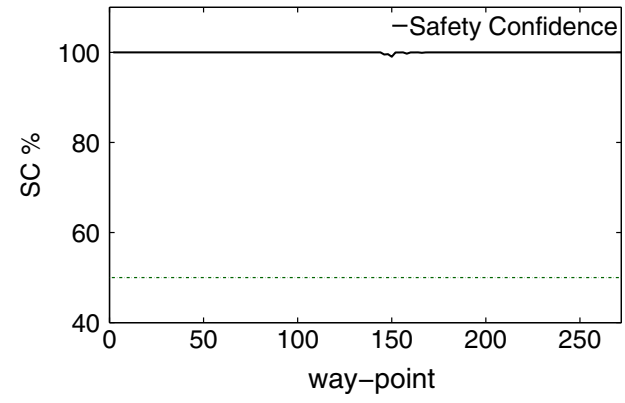

(b) $S C\left(\beta_{\min }=0.20\right)$.

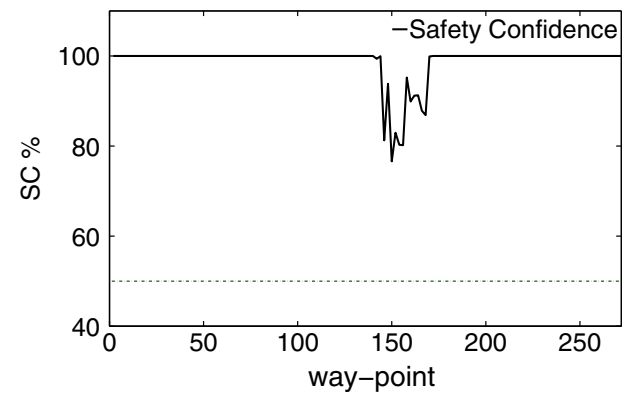

(d) $S C\left(S C_{\min }=70 \%\right)$.

\subsubsection{Results of $A$ * planner in the UTIAS arena}

The UTIAS data is used to study the outcomes of planning longer paths with different values for $\beta_{\min }$ and $S C_{\min }$. Results in Fig. 13 show how when the stability constraint is reasonable medium value, the statistical approach can find more effective and shorter path than deterministic technique (the path shown in orange).

The outcomes of a planner based on different deterministic stability margins are shown in Fig. 13a where the path with lowest allowable safety margin $\beta_{\min }=0.05$ is illustrated in black, and paths with $\beta_{\min }=0.10$ and $\beta_{\min }=0.2$ are depicted in orange and yellow respectively. Gray-scale colour coding indicates height of the terrain from 0 to $2.76 \mathrm{~m}$. A pre-processing algorithm based on terrain gradients was first applied to the model to label out obviously untraversable steep slopes, shown in dark Brown. Given the space limitations, only the uncertainty analysis results of the first two trajectories are shown in Fig. 14, where the mean values of the stability measure using the UT transform at each instant are depicted in red, the standard deviation $\sigma(68 \%)$ and $2 \times \sigma(95 \%)$ around the mean are depicted in dashed red and blue in Fig. 14a, c. Figure 14b, d illustrate the corresponding SC measures.

In the same way Fig. 13b shows the effect of different values of $S C_{\min }$ on the planner, where black, orange and yellow illustrate trajectories with $S C \_$min $=50 \%, S C \_$min $=$
$70 \%$ and SC_min $=90 \%$ respectively. The corresponding uncertainty analysis are shown in Fig. 15.

The result of planning based on the lowest allowable $\beta_{\min }=0.05$ and $S C_{\min }=50 \%$ (depicted in black in Fig. 13a, b respectively) are found quiet coincidental. These two trajectories are going through (A) and passing directly over the central hill $(\mathrm{C})$. Although the planning based on $\beta_{\text {min }}=0.05$ ensures that instant value of $\beta$ are always larger than the minimum value, $\beta_{\mu}$ is found to be negative over the more challenging section, hence resulting in an $S C<50 \%$ i.e. a high risk for a tip-over instability as illustrated in the way-points around 150 in Fig. 14b. This would not represent a dangerous situation when planning is based on an $S C \_$min $=50 \%$ as the planner will reconfigure robot so that it fulfils the minimum safety confidence as illustrated in Fig. 15b. Moreover planning based on more significant stability margins and safety confidence $\left(\beta_{\min }=0.20\right.$ and $S C_{\min }=90 \%$ ) results in longer routes, depicted in yellow in Fig. 13a, b respectively.

Planning based on a comfortable stability margin and safety confidence $\left(\beta_{\min }=0.10\right.$ and $\left.S C_{\min }=70 \%\right)$ produced some interesting results. With $\beta_{\min }=0.10$ the planner could not find a trajectory through the front section (A) and resorted to move up towards (B), eventually finding a path via (D) to the goal. On the other hand, the planner with $S C_{\min }=70 \%$ considered the front section (A) feasible and found a shorter path which goes straight up to the middle of the arena and then coincide with the path with $\beta_{\min }=0.10 \mathrm{in}$ 


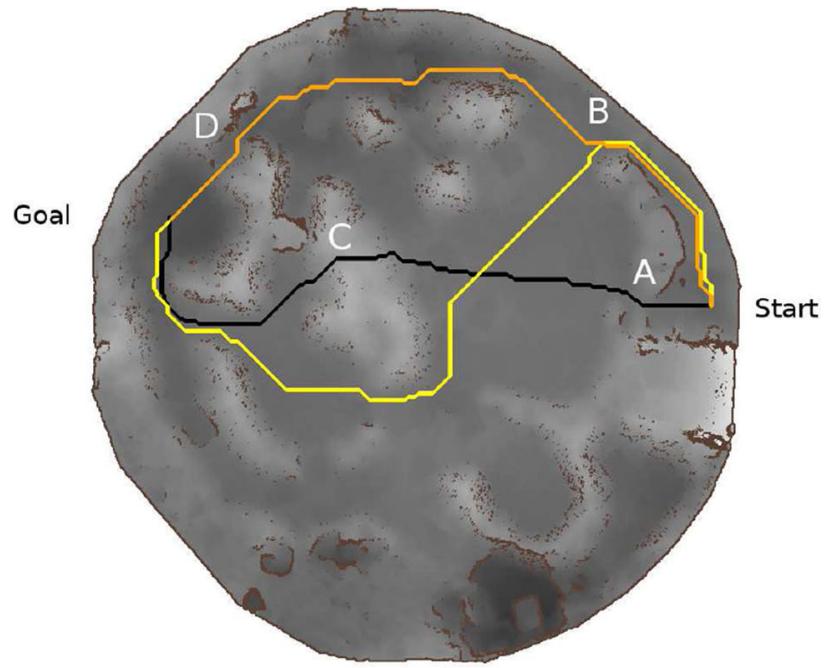

(a) Planning according to $\beta_{\min }$.

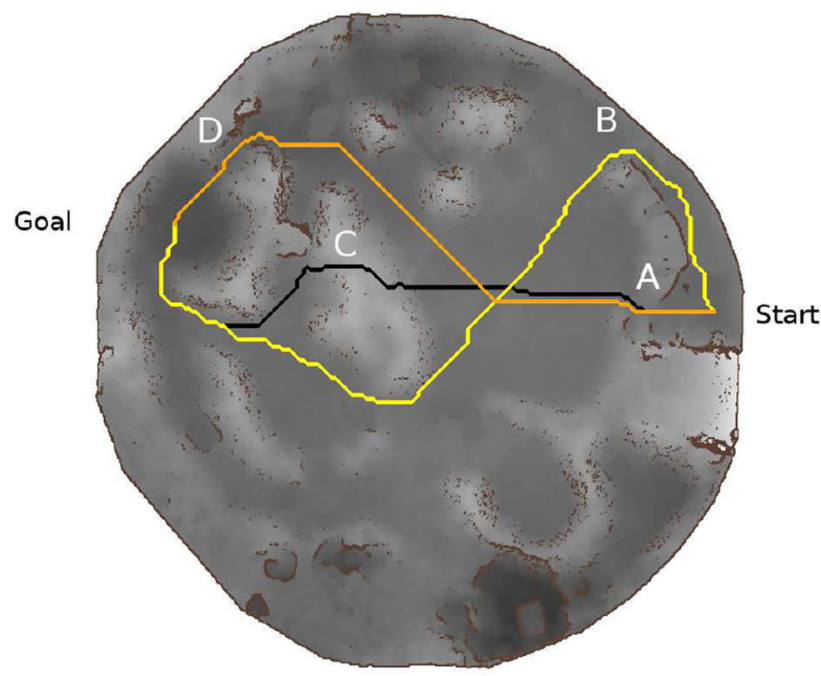

(b) Planning according to $S C_{\text {min }}$.

Fig. 13 Planning in the UTIAS arena. The paths with $\beta_{\text {min }}=0.05$ and $S C_{\text {min }}=50 \%$ are illustrated in black, paths with $\beta_{\text {min }}=0.10$ and $S C_{\min }=70 \%$ are depicted in orange and yellow trajectories showing the paths with $\beta_{\min }=0.20$ and $S C_{\min }=90 \%$ the final stages in the area labelled as (D). Looking at Fig. 15c around way-point 25 it is seen how $\beta$ around (A) is less than $\beta_{\min }=0.10$, revealing the reason why planning based on $\beta_{\min }$ would not consider this area traversable. Looking at the value of $S C$ in Fig. 15d confirms that although $\beta$ is less than $\beta_{\text {min }}=0.10$ around (A), safety confidence is bigger than $70 \%$ and the planner regards this region as comfortably stable to plan over. This example shows how planning based on statistical data instead of the instant values can result in more effective and at the same time safer routes. The overall length of the trajectories illustrated in Fig. 13a, b are summarised in Table 1.
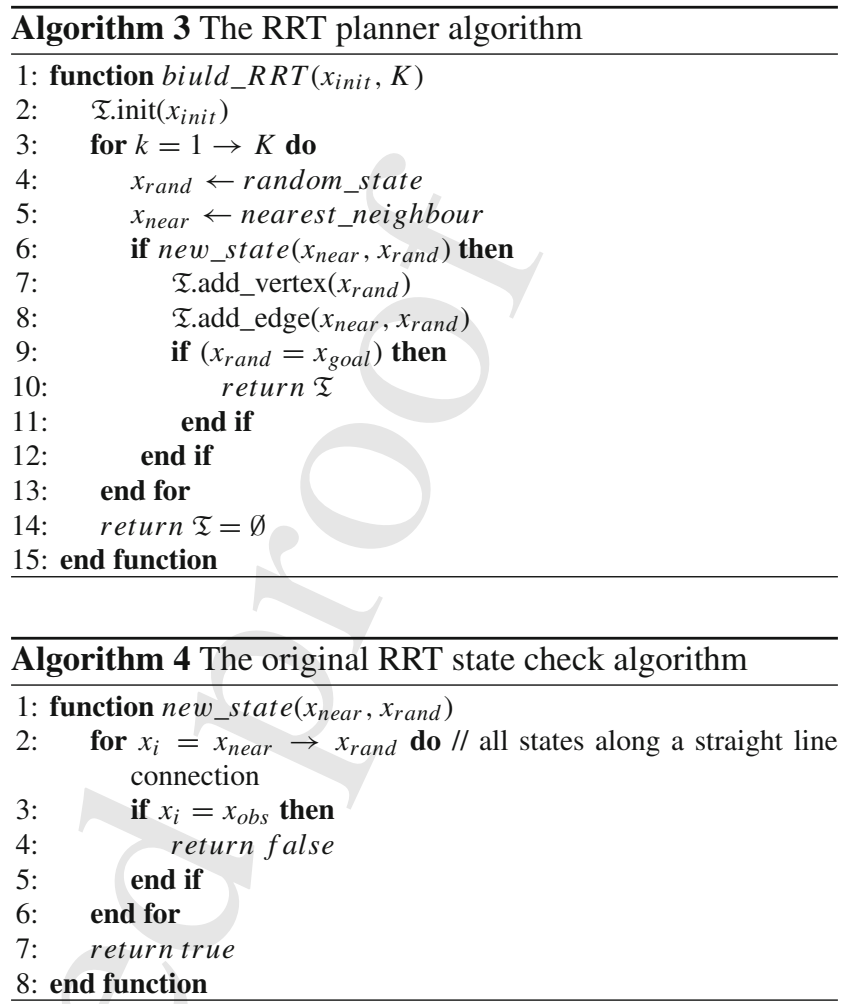

\subsection{Implementation with RRT planner}

In this section an integration of the strategy in the well established sampling based RRT planner is presented for completeness. Fundamentally RRT builds a space-filling tree $(\mathfrak{T})$ and extends it randomly to efficiently search highdimensional spaces. As RRT planners can quickly cover an environment by the random tree expansion, they have been widely used in autonomous robotics path planning. When extending the tree, it is able to regularly check the collision with obstacles and differential constraints (non-holonomic, kino-dynamic etc).

In spite of the fact that the RRT planner does not need a grid to expand, for simplicity and comparison purposes, lets assume that search space is a 2D grid equal to A* algorithm's environment. The grids of the graph are classified into two sets referred to as obstacle and free. The path planning can be viewed as a search in this grid from an initial start node, $x_{\text {init }}$ to the goal node $x_{\text {goal }}$ while avoiding obstacle nodes $x_{o b s}$. An RRT that is rooted at $x_{i n i t}$ and has $K$ vertices can be summarized as an iterative procedure as illustrated in Algorithm 3.

In beginning, the algorithm initiates RRT tree $\mathfrak{T}$ with start node as the first vertex. In each iteration, the algorithm attempts to extend the RRT by adding a random new node $x_{\text {rand }}$. The nearest vertex $x_{\text {near }}$ already in the RRT to the given $x_{\text {rand }}$ will be chosen according to a metric like Euclidean distance. The function new_state is called in 
Fig. 14 Comparison of $S C$ and $\beta$ for the paths depicted in Fig. $13 \mathrm{a}$ in the UTIAS arena, with $\beta_{\min }=0.05$ and $\beta_{\text {min }}=0.10$
Fig. 15 Comparison of $S C$ and $\beta$ over the trajectories depicted in Fig. $13 \mathrm{~b}$ in the UTIAS arena with $S C_{\min }=50 \%$ and $S C_{\min }=70 \%$

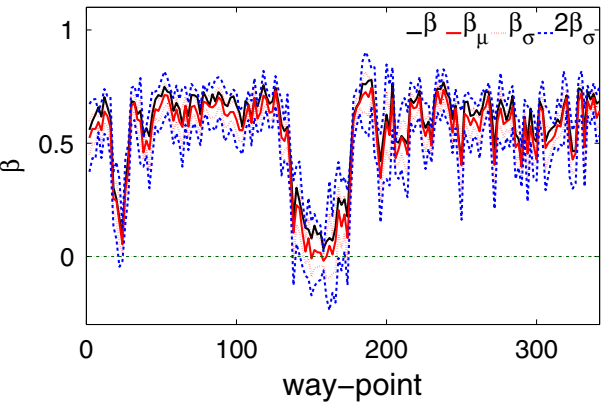

(a) $\beta\left(\beta_{\min }=0.05\right)$.

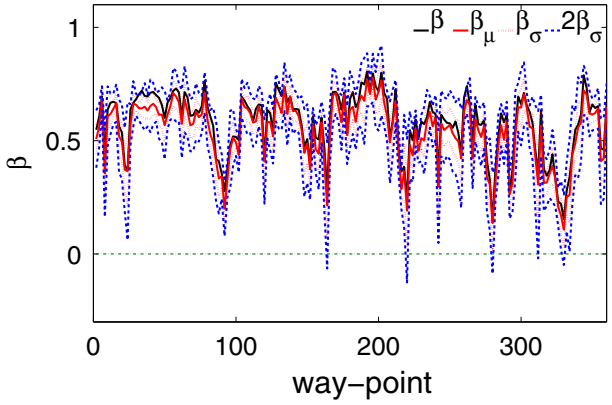

(c) $\beta\left(\beta_{\min }=0.10\right)$.

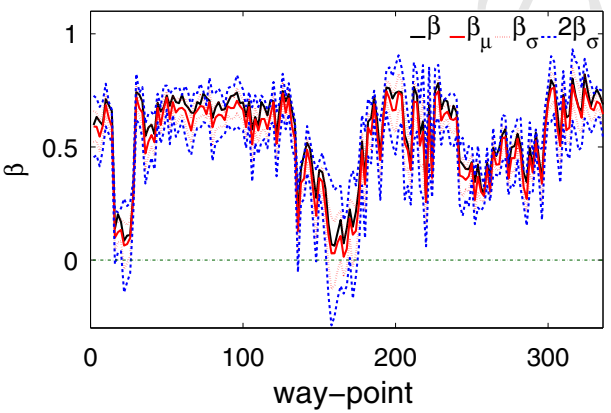

(a) $\beta\left(S C_{\min }=50 \%\right)$.

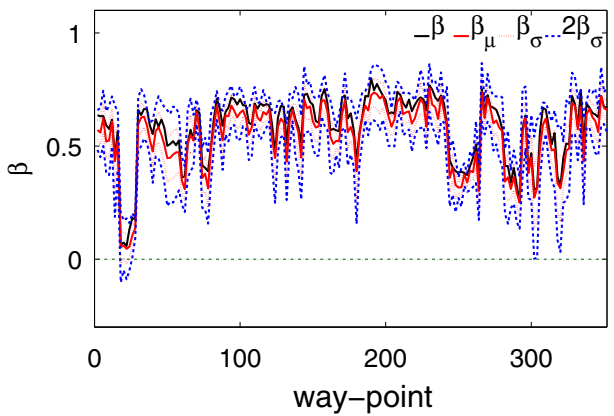

(c) $\beta\left(S C_{\min }=70 \%\right.$.)

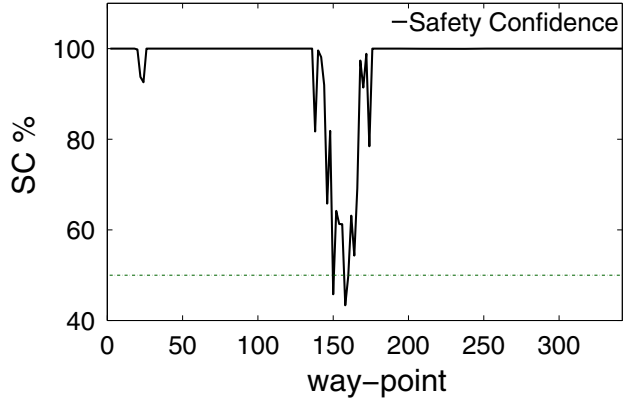

(b) $S C\left(\beta_{\min }=0.05\right)$.

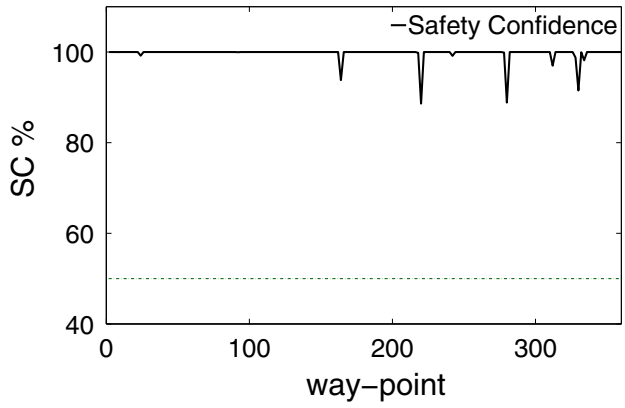

(d) $S C\left(\beta_{\min }=0.10\right)$.

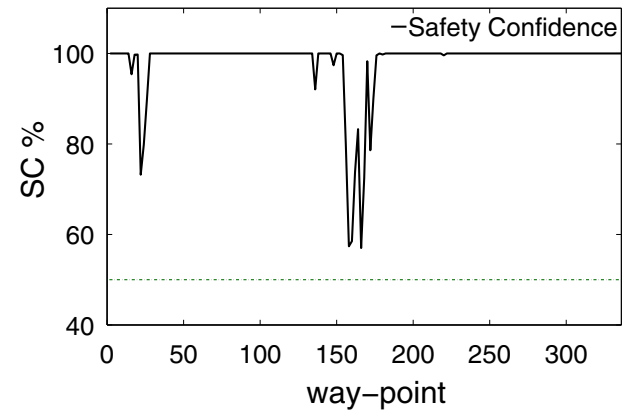

(b) $S C\left(S C_{\min }=50 \%\right)$.

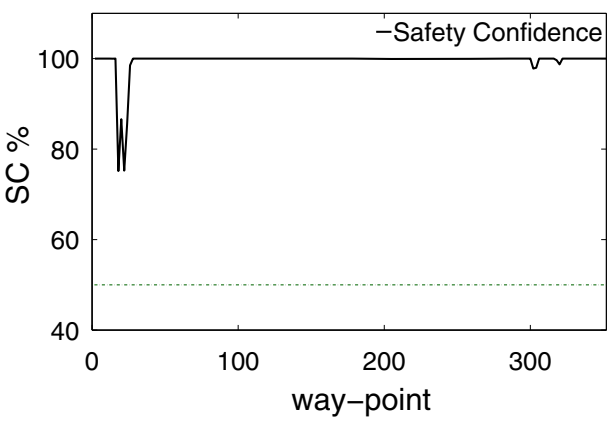

(d) $S C\left(S C_{\min }=70 \%\right.$.) this stage to detect potential collisions to determine whether the $x_{\text {rand }}$ (and all intermediate states) satisfies the global constraints as shown for the simple scenario of obstacle avoidance in Algorithm 4. If new_state is successful, the $x_{\text {rand }}$ is added as a new vertex to $\mathfrak{T}$. An edge from $x_{\text {near }}$ to $x_{\text {rand }}$ is also added. If the recently added vertex reaches the $x_{\text {goal }}$, the algorithm successfully returns $\mathfrak{T}$ and the final path will be the chain of branches from the $x_{\text {goal }}$ back to the $x_{\text {init }}$ node (similar to parent's chain in $\mathrm{A}^{*}$ algorithm). 
Table 1 Overall length of paths shown in Fig. 13a, b

\begin{tabular}{llll}
\hline$\beta_{\min }$ & length $(\mathrm{m})$ & $S C_{\min }$ & length $(\mathrm{m})$ \\
\hline 0.05 & 39.6184 & $50 \%$ & 39.0450 \\
0.10 & 43.4073 & $70 \%$ & 41.8475 \\
0.20 & 54.9470 & $90 \%$ & 53.0440 \\
\hline
\end{tabular}

To guarantee the stability of $\mathfrak{T}$, the new_state function is modified according to Algorithm 5. For each way-point between $x_{\text {near }}$ and $x_{\text {rand }}$, the algorithm calculates the statistical information about the tip-over instance using the ut_transform () function in the 3D physical simulator. The new branch in the RRT tree would be considered safe only if

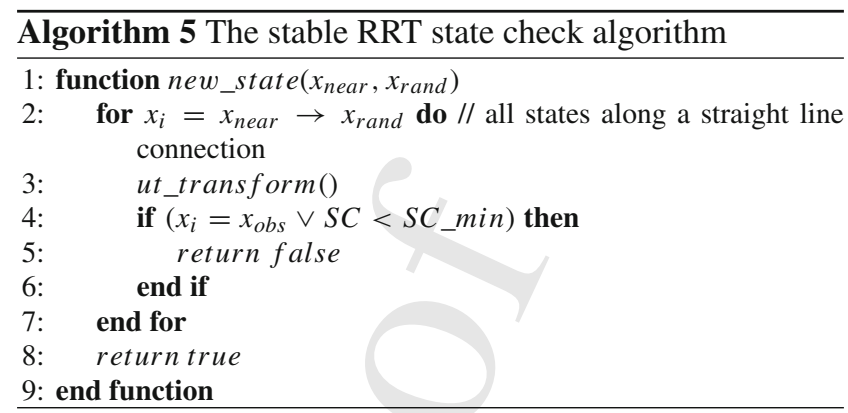

Fig. 16 The block diagram of the stable RRT algorithm

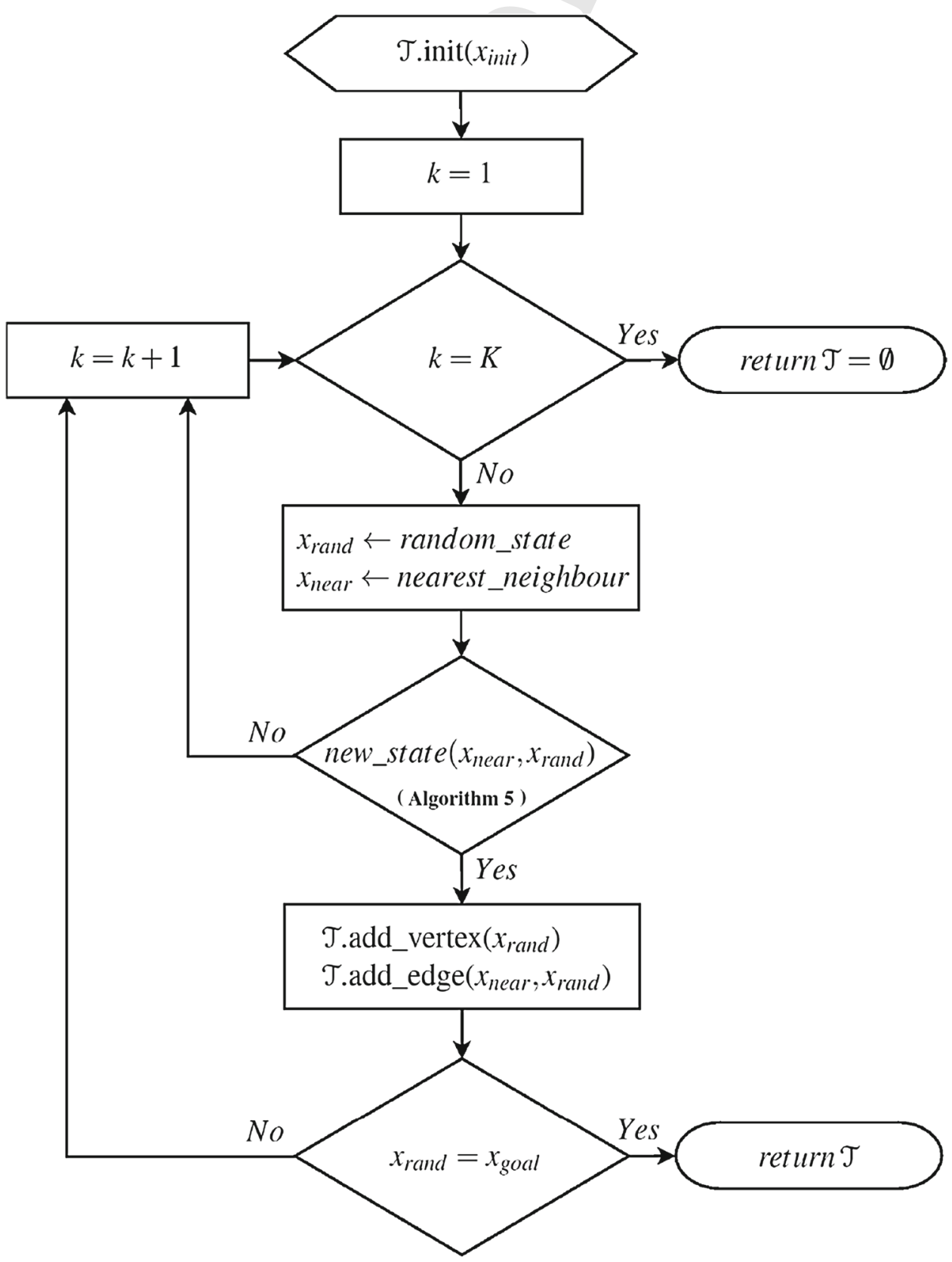

it is collision-free and also satisfies the corresponding minimum safety confidence. The block diagram of the overall stable RRT algorithm is illustrated in Fig. 16. 


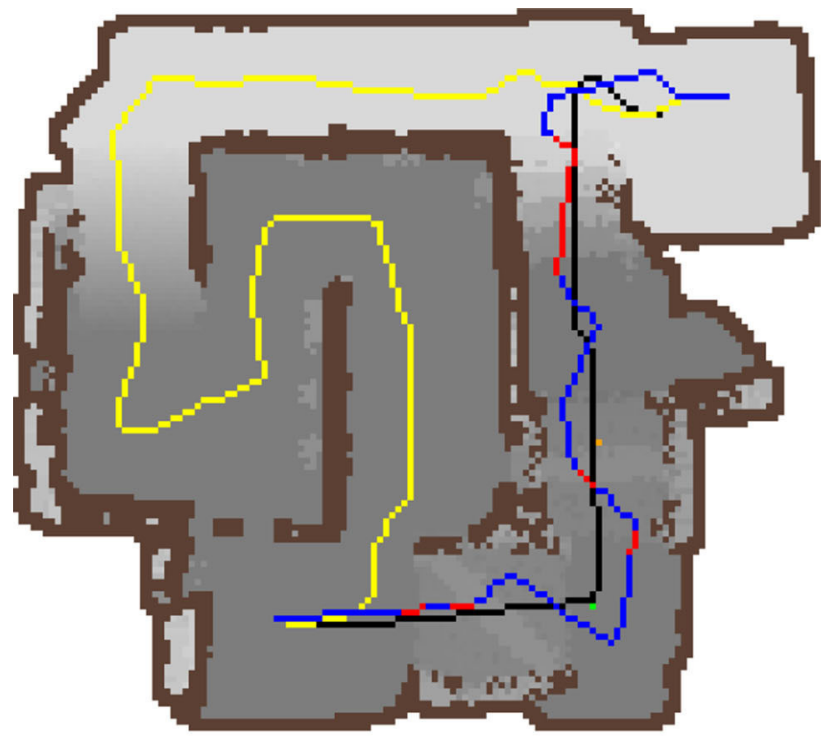

(a) The trajectories from the top view.

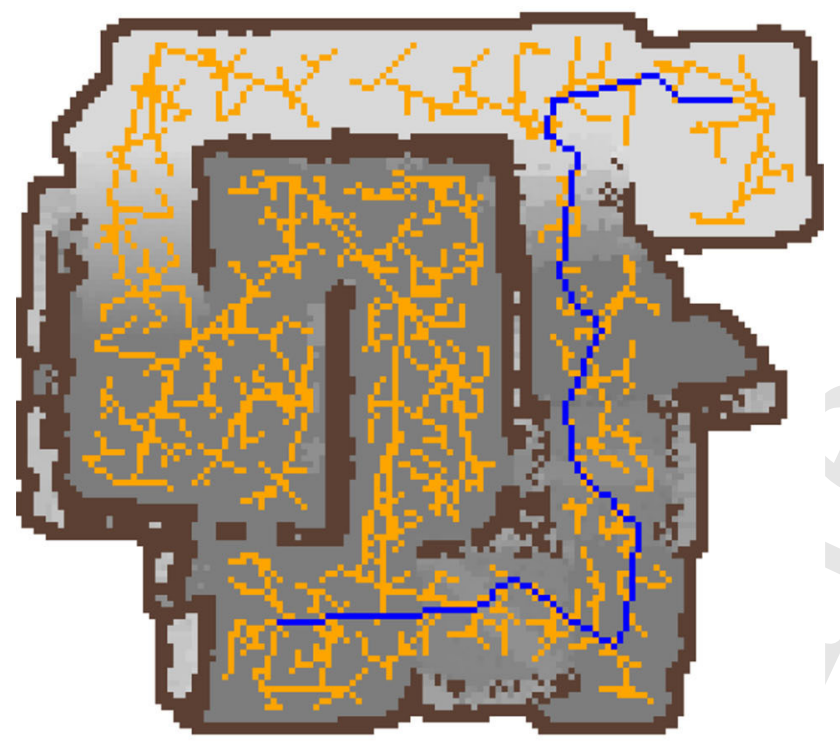

(b) The RRT tree and trajectory of the ordinary path.

Fig. 17 Results of stability criterion in the RRT algorithm in the USAR arena. a Results show the path derived from the original RRT planner in blue with unstable points in red. The stable path with the lowest allowable safety confidence $S C_{\min }=50 \%$ and the trajectories where $S C_{\text {min }}=70 \%$ are depicted in black and yellow respectively. b The RRT tree is depicted in orange and has expanded to almost the entire whole $164 \times 150$ search space. In the majority of times the algorithm came up with the shorter path via stairs as depicted in blue

\subsubsection{Results of RRT planner in the USAR arena}

The preference of planning based on a probabilistic metric in comparison with a deterministic stability measure was discussed in Sect. 4.2. Here we are going to compare the original
RRT planner with the RRT planner constrained on the safety confidence $S C$ measure. Some implementations of RRTs limit the length of the connection between the tree and a new state by a growth factor (Liangjun and Dinesh 2008). This forces the random sample to lie within a maximum distance from the tree and limits the size of the incremental growth. In this work, the random sample is uniformly sampled from the entire search space to allow the tree to quickly expand towards large unsearched areas. This freedom in expansion sometimes results in long straight branches (routes) in the tree, but the algorithm will check the feasibility of all intermediate way-points before accepting the new state.

In the first instance the result of the original RRT is compared with the trajectories achieved from planning based on lowest allowable safety confidence, $S C_{\min }=50 \%$ and a comfortable margin $S C_{\min }=70 \%$, in the USAR arena. The outcomes of the proposed stable RRT planner are illustrated from a top view in Fig. 17 on the USAR arena in comparison with the standard RRT, where Fig. 17a is showing all three trajectories simultaneously, and Fig. 17b presents the RRT tree and trajectory of the ordinary path in a separate figure. A pre-processing algorithm was first applied to the 3D map to determine extreme untraversable areas, e.g. walls and markedly steep slopes. Results in Fig. 17a show the path derived from the original RRT in blue while the way-points where the robot was not stable for the fixed vertical arm and flipper pose are highlighted in red. The stable path with the the lowest allowable safety confidence $S C_{\min }=50 \%$ and the trajectories where $S C_{\text {min }}=70 \%$ are depicted in black and yellow respectively.

While ordinary route and stable path where $S C_{\min }=$ $50 \%$ may find a way to the goal either from stairs or via the ramp in the top left corner of the arena, the planning with more conservative stability constraint of $S C_{\text {min }}=70 \%$ leaves the ramp the only possible trajectory. As illustrated in Fig. 17b, the original RRT tree has expanded entire the USAR arena, but most of the time the shorter route via the stairs was chosen as the final trajectory.

The robot configurations along stable trajectories are depicted in Fig. 18, where Fig. 18a, b illustrate the outcomes of the stable paths where $S C_{\min }=50 \%$ and $S C_{\min }=70 \%$ respectively. Only a limited number of the robot poses are shown for clarity. The corresponding uncertainty analysis are shown in Fig. 19. Both planners have handled the corresponding $S C_{\min }$ constraint successfully while expanding the RRT trees. To fulfil $S C_{\min }=50 \%$, the planner has configured robot to $\phi_{a}=0^{\circ}$ over the stairs section depicted in Fig. 18a, while given the higher certainty of the map over the ramp, the algorithm can satisfy the stability constraint $S C_{\min }=70 \%$ with a better field of view configuration $\left(\phi_{a}=50^{\circ}\right)$, as illustrated in Fig. 18b.

Table 2 summarises the statistical information about average length and standard deviation $(\sigma)$ of RRT paths over 


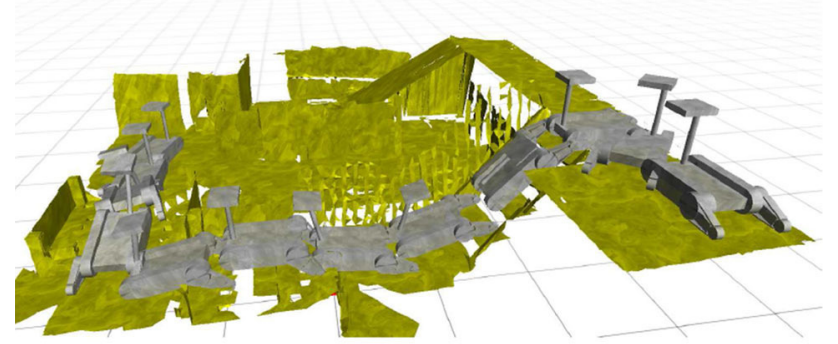

(a) Path where SC_min $=50 \%$.

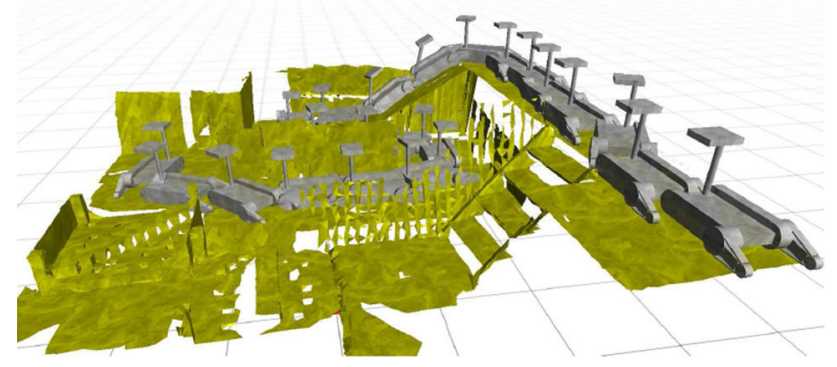

(b) Path where $S C \_$min $=70 \%$.

Fig. 18 Results of probabilistic stability criterion on RRT algorithm in the USAR arena

Fig. 19 Comparison of $S C$ and $\beta$ over the trajectories depicted in Fig. 18 in the USAR arena. The horizontal dark green dash-dot lines are indicating the reference points where $\beta=0$ or $S C_{\text {min }}=50 \%$
Table 2 Comparison of average length and $\sigma$ of RRT paths in 10 runs versus the corresponding minimum $\mathrm{A} *$ trajectories in the USAR arena

\begin{tabular}{lccl}
\hline Path $(\mathrm{m})$ & Minimum $\left(\mathrm{A}^{*}\right)$ & Average RRT & $\sigma$ \\
\hline Original & 7.9899 & 9.5692 & 1.7060 \\
$S C_{\text {min }}=50 \%$ & 8.2485 & 9.8189 & 1.6117 \\
$S C_{\text {min }}=70 \%$ & 14.0727 & 17.2135 & 1.3460 \\
\hline
\end{tabular}

of test runs $\sigma$ will generally decrease as $S C_{\min }$ increases. It can be observed how for the original RRT and the case when $S C_{\min }=50 \%, \sigma$ values are close together and reasonably larger than the RRT where $S C_{\min }=70 \%$. This is because the first two planners have, independently of the adopted configurations, two clear alternatives when it comes to traverse the terrain to go to the goal point, through a ramp or through the stairs, whereas the RRT where $S C_{\min }=70 \%$ leaves the ramp as the only possible trajectory. This behaviour will become more apparent in the results of the UTIAS arena as the planner would have a larger search space.

\subsubsection{Results of RRT planner in the UTIAS arena}

As the UTIAS terrain mimics an outdoor environment, the comfortable stability confidence is increased to $S C_{\min }=$ $90 \%$ when searching for a reliable tip-over margin. In the same way, Fig. 20 shows the result of the stable RRT algorithm in the UTIAS arena, where all three trajectories are
835 836 837 838 839 840 841 842 843 844 845 846

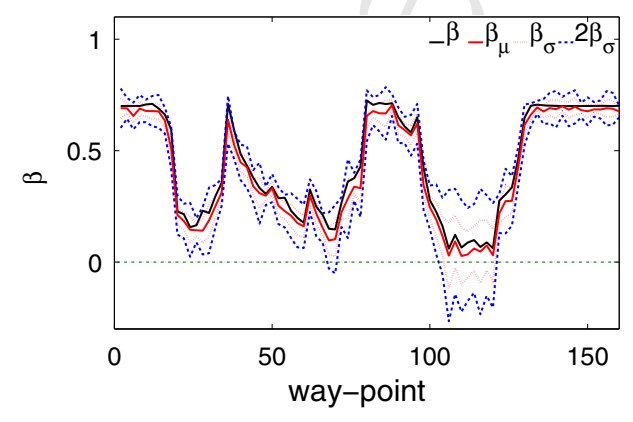

(a) $\beta\left(S C_{\min }=50 \%\right)$.

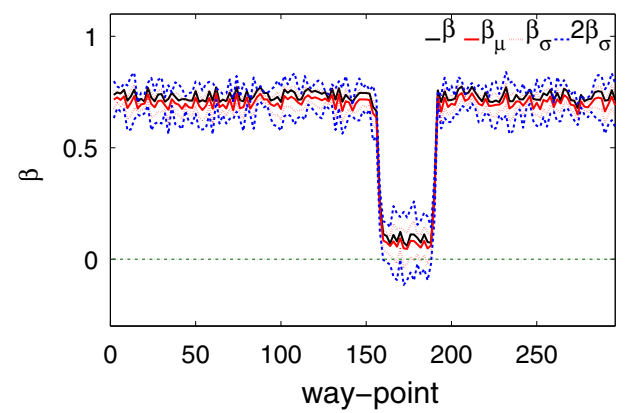

(c) $\beta\left(S C_{\min }=70 \%\right)$.

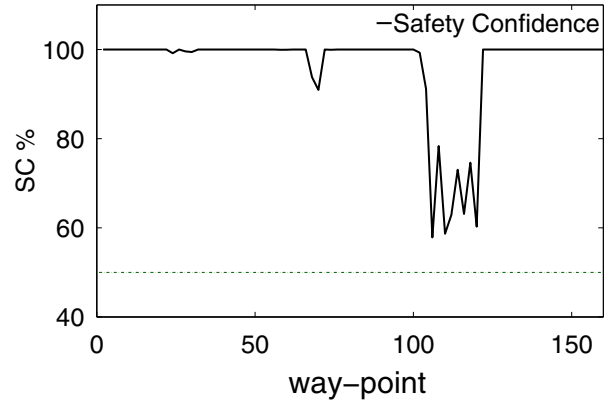

(b) $S C\left(S C_{\min }=50 \%\right)$.

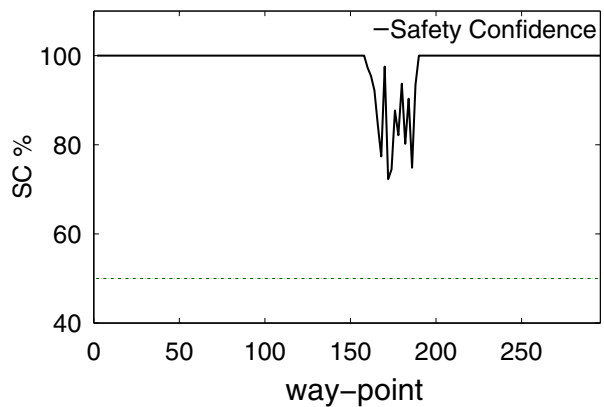

(d) $S C\left(S C_{\min }=70 \%\right)$. 


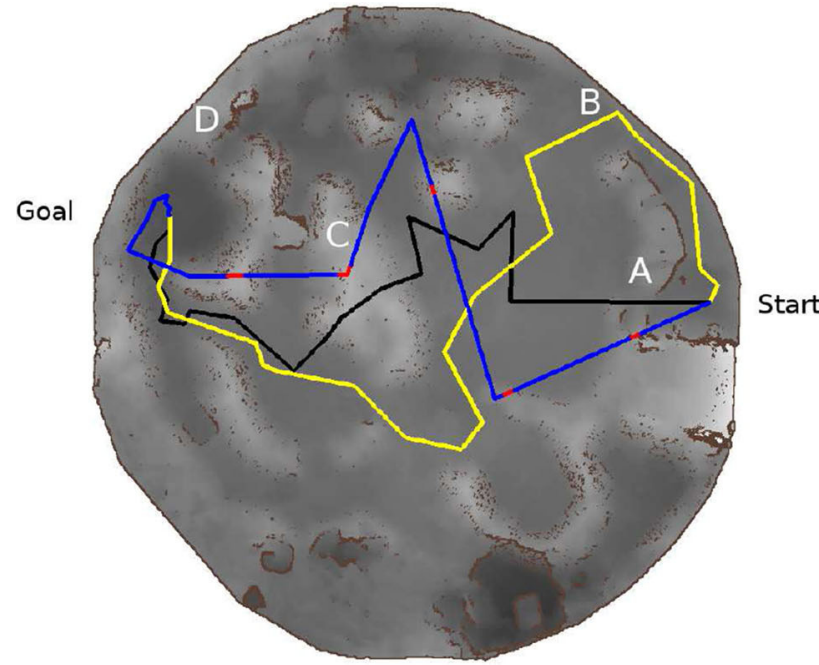

(a) The trajectories from the top view.

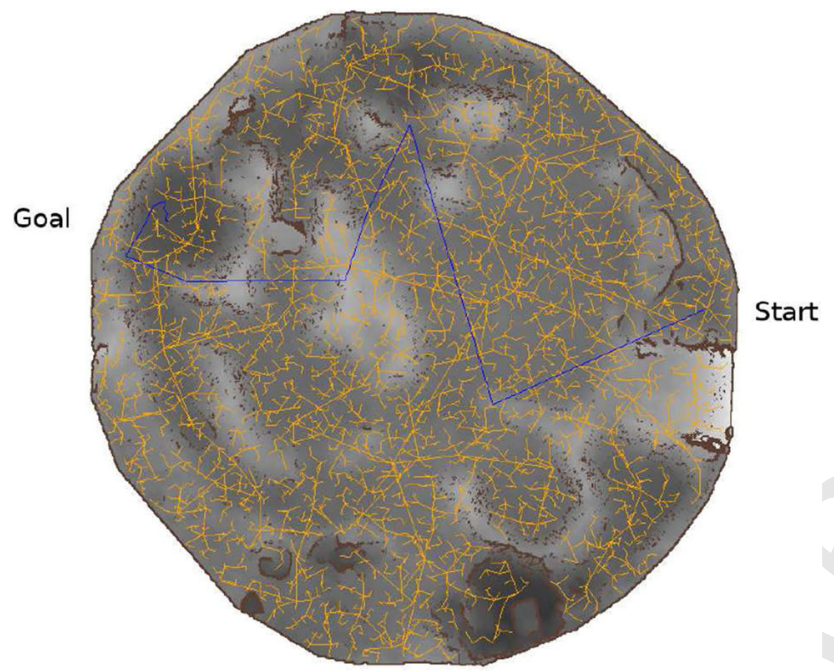

(b) The RRT tree and trajectory of the ordinary path.

Fig. 20 Results of stability criterion in the RRT algorithm in the UTIAS arena. a Results show the path derived from the original RRT planner in blue with unstable points in red. The stable path with the lowest allowable safety confidence $S C_{\min }=50 \%$ and the trajectories where $S C_{\min }=90 \%$ are depicted in black and yellow respectively. b The RRT tree is depicted in orange and has expanded to almost the entire whole $784 \times 776$ search space. In most instances the planner came up with a route via $(A)$ and in this example eventually found a path crossing from $(C)$ to the goal

depicted in Fig. 20a for comparison and Fig. 20b is separately illustrating the expansion of the RRT tree and resulting trajectory for the original planner. Figure 20a pictures the original RRT path in blue (with unstable points in red) and compares the effect of different values of $S C_{\min }$ on the planner, where black and yellow illustrate trajectories where $S C \_$min $=50 \%$ and SC_min $=90 \%$ respectively.

While ordinary and stable RRT planner where $S C_{\min }=$ $50 \%$ may find a way to the goal either through (A) or (B), the planning with the highly conservative stability constraint of $S C_{\min }=90 \%$ can only go through (B). As illustrated in Fig. 20b, the original RRT tree has expanded the entire UTIAS arena as well, but mostly the planner came up with a route via (A) and, in this example, eventually found a path crossing from $(\mathrm{C})$ to the goal. In the trials provided in Fig. 20a, the stable path where $S C_{\min }=50 \%$ is going through (A) and passing directly over the central hill (C), while the more conservative path where $S C_{\min }=90 \%$ avoids both of these regions and moves up towards (B) choosing the longest and safest route which goes around part (C). The corresponding uncertainty analysis for stable routes are shown in Fig. 21. According to this figure, the $S C_{\min }$ over the resulting path and entire RRT tree was effectively satisfied while searching the space for more branches.

In the same way, the statistical information about average length and $\sigma$ of the paths are collected in Table 3. As expected from the previous observations in the USAR arena, $\sigma$ is continuously descending as more constraints are applied to the planners. Yet given the larger path planning search space in the outdoor terrain when compared to the more restrictive mock-up indoor arena, the relative $\sigma$ of the routes in the UTIAS arena are significantly larger than their USAR arena counterparts.

\section{Conclusions and discussion}

This article presents a probabilistic approach to account for robot's stability uncertainty when planning motions over uneven terrains. The proposed algorithm can exploit information gained from a statistical stability analysis to plan safe and effective routes under the presence of uncertainty in the robot kinematics, terrain model and localisation on the terrain. The integration of the strategy with two well studied grid based and sampling based algorithms i.e. $\mathrm{A}^{*}$ and RRT planners, is presented.

Simulation results in an indoor rescue arena and an outdoor rover testing facility demonstrate the advantages of planning based on statistical stability information when compared with a deterministic approach. The results of path planning based on the lowest allowable safety margin shows that by setting an arbitrary lower boundary, the deterministic planner's limited concern about the instantaneous value of $\beta$ results in paths with instances where, although $\beta$ is computed to be always above a certain $\beta_{\min }$, the corresponding $\beta_{\mu}$ can actually become negative ( $S C<50 \%$ ) at times, indicating an unacceptable high risk of tip-over instability. The contingency of this potentially dangerous situation is minimised when planning is carried out based on SC_min, as the planner will reconfigure the robot so that it fulfils the minimum safety confidence at any given time. Moreover, when uncertainty levels are small (on ramps or sloped areas for 
Fig. 21 Comparison of $S C$ and $\beta$ over the trajectories depicted in Fig. 20 in the UTIAS arena. The horizontal dark green dash-dot lines are indicating the reference points where $\beta=0$ or $S C_{\min }=50 \%$

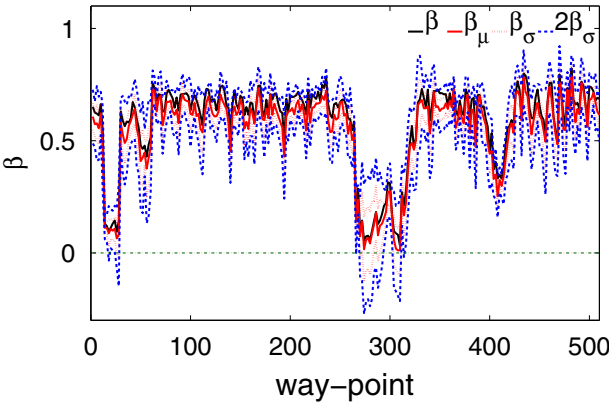

(a) $\beta\left(S C_{\min }=50 \%\right)$.

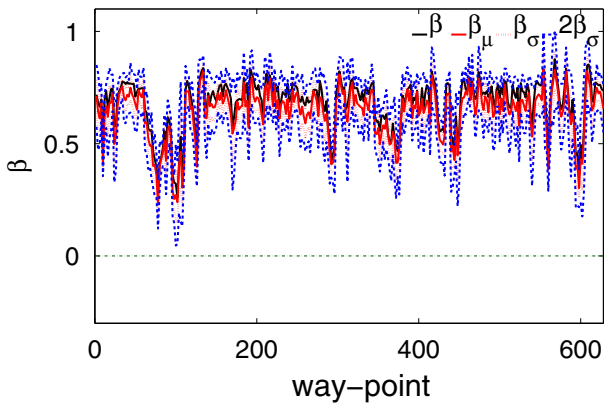

(c) $\beta\left(S C_{\min }=90 \%\right)$.

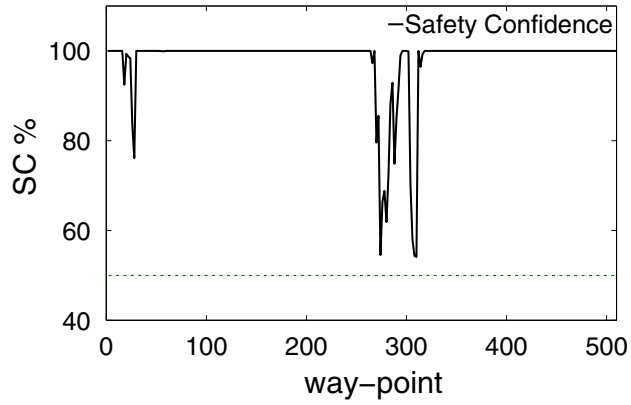

(b) $S C\left(S C_{\min }=50 \%\right)$.

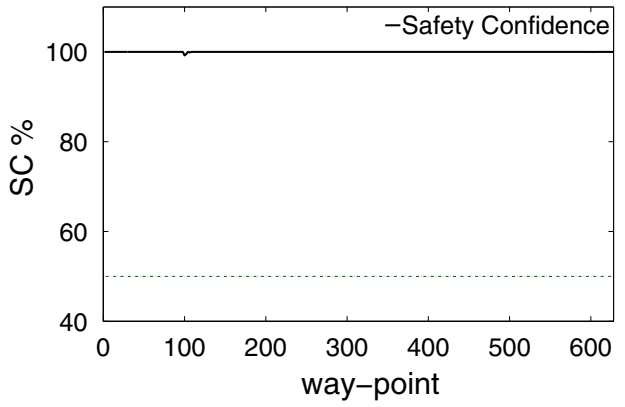

(d) $S C\left(S C_{\min }=90 \%\right)$.

Table 3 Comparison of average length and $\sigma$ of RRT paths in 10 runs versus the corresponding minimum $\mathrm{A}^{*}$ trajectories in the UTIAS arena

\begin{tabular}{lllr}
\hline Path $(\mathrm{m})$ & Minimum $\left(\mathrm{A}^{*}\right)$ & Average RRT & \multicolumn{1}{l}{$\sigma$} \\
\hline Original & 33.0823 & 56.5629 & 15.3264 \\
$S C_{\text {min }}=50 \%$ & 39.0450 & 59.6210 & 11.3975 \\
$S C_{\text {min }}=90 \%$ & 53.0440 & 73.2896 & 7.3383 \\
\hline
\end{tabular}

instance) the probabilistic approach is able to exploit this to generate postures with better visibility than the deterministic planner. Comparison of the resulting trajectories in the outdoor UTIAS arena shows planning based on the proposed statistical stability methodology can result in more effective, and at the same time, safer routes.

The proposed scheme relies on a physics engine (e.g. ODE) and surrounding terrain information to derive probabilistic stable paths. Despite the computational advances that UT transform brings when compared to SMC in dealing with uncertainty modelling, processing time remains considerable, particularly as it increases with the size of the 3D mesh (Smith 2005). In showing the validity of the proposed approach there was limited need to endeavour planning in real-time, however it is anticipated that employing a dedicated graphics processing unit for the surface manipulation and physics simulations required to derive probabilistic stable paths would significantly improve the processing time to the point of making it altogether viable for modest sizes in exploratory settings.
While the probabilistic stable motion planning strategy has been shown here for the more generalised case of reconfigurable robots, it is naturally equally applicable for fixed-configuration robots where stability margins will dictate safer routes to traverse under the assumption of lesser DoF's, hence simply a reduced grid search space given the lack of ability to assume other poses.

\section{References}

Berg, J. V. D., Abbeel, P., \& Goldberg, K. (2011). Lqg-mp: Optimized path planning for robots with motion uncertainty and imperfect state information. The International Journal of Robotics Research, 30(7), 895-913.

Besseron, G., Grand, C., Amar, F.B., \& Bidaud, P. (2008). Decoupled control of the high mobility robot hylos based on a dynamic stability margin. In Proceedings of IEEE/RSJ International Conference on Intelligent Robots and Systems (pp. 22-23). Nice, France.

Brooks, A., Makarenko, A., Williams, S., \& Durrant-Whyte, H. (2006). Parametric POMDPs for planning in continuous state spaces. Robotics and Autonomous Systems, 54(11), 887-897.

Candido, S., Davidson, J., \& Hutchinson, S. (2010). Exploiting domain knowledge in planning for uncertain robot systems modeled as pomdps. In Proceedings of IEEE International Conference on Robotics and Automation (pp. 3596-3603). Alaska, USA.

Coelho, P., \& Nunes, U. (2005). Path-following control of mobile robots in presence of uncertainties. IEEE Transactions on Robotics, 21(2), 252-261.

Freitas, G., Gleizer, G., Lizarralde, F., \& Hsu, L. (2010). Kinematic reconfigurability control for an environmental mobile robot oper- 
ating in the amazon rain forest. Journal of Field Robotics, 27(2), 197-216.

Gill, R., Kulic, D., \& Nielsen, C. (2013). Robust path following for robot manipulators. In Proceedings of IEEE/RSJ International Conference on Intelligent Robots and Systems (pp. 3412-3418). Tokyo, Japan.

Greenberg, M. (1998). Advanced engineering mathematics, 2/E. New Delhi: Pearson Education India.

Hart, P. E., Nilsson, N. J., \& Raphael, B. (1968). A formal basis for the heuristic determination of minimum cost paths. IEEE Transactions on Systems Science and Cybernetics, 4(2), 100-107.

Iagnemma, K., \& Dubowsky, S. (2004). Mobile robot in rough terrain (Vol. 12). Berlin: Springer Tracts in Advanced Robotics.

Iagnemma, K., Rzepniewski, A., Dubowsky, S., \& Schenker, P. (2003). Control of robotic vehicles with actively articulated suspensions in rough terrain. Autonomous Robots, 14(1), 5-16.

Julier, S., \& Uhlmann, J. (2004). Unscented filtering and nonlinear estimation. Proceedings of the IEEE, 92(3), 401-422.

Keiji, N., Seiga, K., Yoshito, O., Kazuki, O., Kazuya, Y., Satoshi, T., et al. (2013). Emergency response to the nuclear accident at the fukushima daiichi nuclear power plants using mobile rescue robots. Journal of Field Robotics, 30(1), 44-63.

LaValle, S. (1998). Rapidly-exploring random trees: A new tool for path planning. Technical report, Iowa State University, Dept. of Computer Science.

LaValle, S. (2006). Planning algorithms. Cambridge: Cambridge University Press.

Liang, D., Zongquan, D., Haibo, G., Junlong, G., Dapeng, Z., \& Karl, I. (2013). Experimental study and analysis of the wheels' steering mechanics for planetary exploration wheeled mobile robots moving on deformable terrain. The International Journal of Robotics Research, 32(6), 712-743.

Liangjun, Z., \& Dinesh, M. (2008). An efficient retraction-based rrt planner. In Proceedings of IEEE International Conference on Robotics and Automation (pp. 3743-3750). Pasadena, USA.

Liu, Y., \& Liu, G. (2010). Interaction analysis and online tip-over avoidance for a reconfigurable tracked mobile modular manipulator negotiating slopes. IEEE/ASME Transactions on Mechatronics, 15(4), 623-635.

Matthijs, S., \& Nikos, V. (2005). Perseus: Randomized point-based value iteration for pomdps. Journal of Artificial Intelligence Research, 24, 195-220.

Norouzi, M., Miro, J.V., \& Dissanayake, G. (2013a). Planning stable and efficient paths for articulated mobile robots on challenging terrains. In Proceedings of Australasian Conference on Robotics and Automation (p. 10). Sydney, Australia: UNSW.

Norouzi, M., Miro, J.V., \& Dissanayake, G. (2013b). A statistical approach for uncertain stability analysis of mobile robots. In Proceedings of IEEE International Conference on Robotics and Automation (pp. 191-196). Karlsruhe, Germany.

Norouzi, M., Miro, J.V., Dissanayake, G., \& Vidal-Calleja, T. (2014). Path planning with stability uncertainty for articulated mobile vehicles in challenging environments. In IEEE/RSJ Proceedings of International Conference on Intelligent Robots and Systems (pp. 1748-1753). Chicago, Illinois, USA.

Papadopoulos, E., \& Rey, D. (1996). A new measure of tipover stability margin for mobile manipulators. In Proceedings of IEEE International Conference on Robotics and Automation (vol. 4, pp. 3111-3116). Minneapolis, USA.

Papadopoulos, E., \& Rey, D. (2000). The force angle measure of tipover stability margin for mobile manipulatiors. Vehicle System Dynamics, 33(1), 29-48.

Parks, P. (1966). Liapunov redesign of model reference adaptive control systems. IEEE Transactions on Automatic Control, 11(3), 362367 .
Roan, P., Burmeister, A., Rahimi, A., Holz, K., \& Hooper, D. (2010). Real-world validation of three tipover algorithms for mobile robots. In Proceedings of IEEE International Conference on Robotics and Automation (pp. 4431-4436). Anchorage, Alaska, USA.

Rubinstein, \& Reuven, (1981). Simulation and the Monte Carlo method. New York: Wiley.

Santosh, H., Heijden, V. D., Wam, G., Frits, V. E., Alfred, S., \& Cajo, T. B. (2014). Laser range finder model for autonomous navigation of a robot in a maize field using a particle filter. Computers and Electronics in Agriculture, 100, 41-50.

Sebastian, T., Wolfram, B., \& Dieter, F. (2005). Probabilistic robotics. Cambridge: MIT Press.

SeungBeum, S., JunHo, C., ChangHyun, C., YeonSub, J., Seung-Yeup, H., \& Sungchul, K. (2014). Mine detecting robot system. In Field and Service Robotics (pp. 97-109). Springer.

Siegwart, R., \& Nourbakhsh, I. R. (2004). Introduction to autonomous mobile robotos. Cambridge: The MIT press.

Smith, R. (2005). Open dynamics engine. http://www.ode.org/.

Toit, N. D., \& Burdick, J. (2012). Robot motion planning in dynamic, uncertain environments. IEEE Transactions on Robotics, 28(1), 101-115.

Tong, C. H., Gingras, D., Larose, K., Barfoot, T. D., \& Dupuis, E. (2013). The canadian planetary emulation terrain 3D mapping dataset. The International Journal of Robotics Research, 32(4), 389-395.

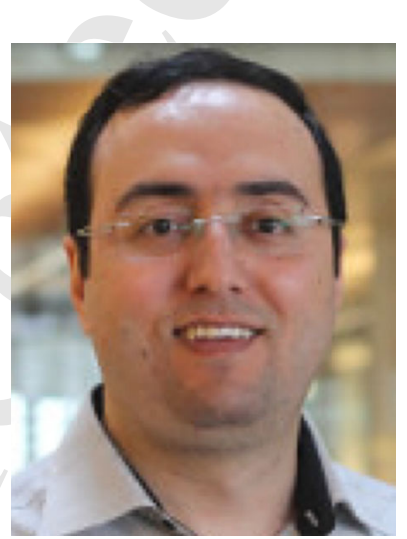

Mohammad Norouzi received his B.Sc. and M.Sc. degrees in Electrical Engineering, from the Qazvin Branch, Islamic Azad University (QIAU) and Iran University of Science and Technology, Iran, in 2003 and 2006 respectively. He received his Ph.D. degree from the University of Technology, Sydney (UTS), Australia, in 2014. He is currently a Assistant Professor with the Department of Electrical, Biomedical andMechatronics Engineering, and a researcher with the Mechatronics Research Lab (MRL), at QIAU. His research area includes autonomous mobile robot navigation and mapping, humanrobot interaction and mechatronics systems.

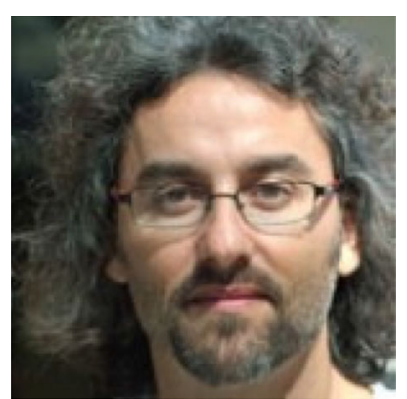

Jaime Valls Miro (Valencia, Spain, 1-11-1969) received his B.Eng. and M.Eng. degrees in Computer Science Systems, from the Valencia Polytechnic University, Spain, in 1990 and 1993 respectively. He received his $\mathrm{PhD}$ degree from Middlesex University, England, in 1998. His thesis examined the use of dynamics for trajectory planning and optimal control of industrialmanipulators. Before joining the Centre for Autonomous Systems at the University of Technology Sydney, he worked for 5 years as a software and control systems analyst in the underwater robotics industry for a London-based company. Currently, he is an Associate Professor with the Centre for Intelligent Mechatronic Systems (CIMS) at UTS. His 
research activities include autonomous mobile robot navigation and mapping (in particular in unstructured scenarios such asUSAR), visual SLAM, assistive robotics, machine learning and human-robot interaction. He is a regular reviewer of scientific works published in the top international robotic journals and conferences.

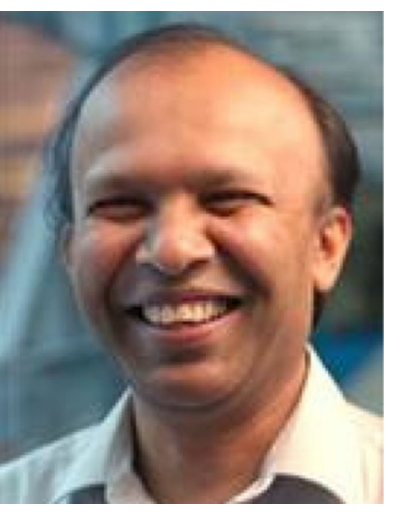

Gamini Dissanayake is the James N Kirby Professor of Mechanical and Mechatronic Engineering at University of Technology, Sydney (UTS). He leads the UTS Centre for Autonomous System; a team of fifty staff and students working in Robotics. He graduated in Mechanical and Production Engineering from the University of Peradeniya, Sri Lanka. He received his M.Sc. in Machine Tool Technology and Ph.D in Mechanical Engineering from the University of Birmingham, England. He taught at University of Peradeniya, Sri Lanka, National University of Singapore and University of Sydney before joining UTS in 2002. His team has an extensive track record of industry collaboration on a range of robotic systems with partners including NSW Roads and Maritime Services, Patrick Technology, Pempek Systems, Burwell Technology, Meat and Livestock Australia and Sydney Water.
1107 1108 1109 1110 1111 